\title{
Influence of Excipients on Cocrystal Stability and Formation
}

\author{
Marwah Aljohani, ${ }^{1}$ Patrick McArdle,,${ }^{1,2, *}$ and Andrea Erxleben ${ }^{1,2, *}$ \\ ${ }^{1}$ School of Chemistry, National University of Ireland, Galway, Ireland \\ ${ }^{2}$ Synthesis and Solid State Pharmaceutical Centre (SSPC), Ireland \\ *Corresponding author email address: andrea.erxleben@nuigalway.ie (AE); \\ p.mcardle@nuigalway.ie (PM)
}

\section{Supporting Information}


Table S1. Preparation of the cocrystals by ball milling. Weights of ctz and coformer. ${ }^{\text {a }}$

\begin{tabular}{|l|l|l|}
\hline cocrystal & mg ctz & mg coformer \\
\hline ctz-bipy & 328.2 & 86.6 \\
\hline ctz-ebipy & 310.5 & 191.3 \\
\hline ctz-pbipy & 295.7 & 198.2 \\
\hline ctz-pyr & 393.3 & 106.5 \\
\hline ctz-hyp & 378.5 & 121.7 \\
\hline ctz-hma & 340.0 & 161.2 \\
\hline ctz-bza & 354.8 & 145.3 \\
\hline ctz-ina & 354.8 & 146.5 \\
\hline ctz-nia & 354.8 & 146.4 \\
\hline ctz-cbz & 277.9 & 444.1 \\
\hline ctz-aca & 416.9 & 83.2 \\
\hline ctz-ppa & 402.1 & 99.4 \\
\hline (bzamH $\left.{ }^{+}\right)\left(\right.$ctz $\left.^{-}\right)$ & 354.8 & 144.1 \\
\hline htz-nia & 178.2 & $73.2^{b}$ \\
\hline
\end{tabular}

${ }^{\text {a }}$ To each sample $50 \mu \mathrm{L}$ acetonitrile was added. ${ }^{\mathrm{b}}$ Instead of acetonitrile, $50 \mu \mathrm{L}$ methanol was used.

Table S2. Milling of the cocrystals with excipients. Composition of samples.

\begin{tabular}{|l|l|l|}
\hline sample & mg cocrystal & mg excipient \\
\hline ctz-bipy/PVP & 414.8 & 328.2 \\
\hline ctz-bipy/MCC & 414.8 & 328.2 \\
\hline ctz-ebipy/PVP & 501.8 & 310.5 \\
\hline ctz-ebipy/MCC & 501.8 & 310.5 \\
\hline ctz-pbipy/PVP & 493.9 & 295.7 \\
\hline ctz-pbipy/MCC & 493.9 & 295.7 \\
\hline ctz-pyr/PVP & 499.8 & 393.3 \\
\hline ctz-pyr/MCC & 499.8 & 393.3 \\
\hline ctz-hyp/PVP & 500.2 & 378.5 \\
\hline ctz-hyp/MCC & 500.2 & 378.5 \\
\hline ctz-hma/PVP & 501.2 & 340.0 \\
\hline ctz-hma/MCC & 501.2 & 340.0 \\
\hline ctz-bza/PVP & 500.1 & 354.8 \\
\hline ctz-bza/MCC & 500.1 & 354.8 \\
\hline ctz-ina/PVP & 501.3 & 354.8 \\
\hline ctz-ina/MCC & 501.3 & 354.8 \\
\hline ctz-nia/PVP & 501.2 & 354.8 \\
\hline ctz-nia/MCC & 501.2 & 354.8 \\
\hline ctz-nia/HPC & 501.2 & 354.8 \\
\hline ctz-nia/ $\alpha-$ lactose & 501.2 & 354.8 \\
\hline ctz-nia/NaTC & 501.2 & 354.8 \\
\hline ctz-nia/DA & 501.2 & 354.8 \\
\hline
\end{tabular}




\begin{tabular}{|c|c|c|}
\hline ctz-cbz/PVP & 722.0 & 277.9 \\
\hline ctz-cbz/MCC & 722.0 & 277.9 \\
\hline ctz-cbz/HPC & 722.0 & 277.9 \\
\hline ctz-cbz/ $\alpha$-lactose & 722.0 & 277.9 \\
\hline ctz-cbz/NaTC & 722.0 & 277.9 \\
\hline ctz-cbz/DA & 722.0 & 277.9 \\
\hline ctz-aca/PVP & 500.1 & 416.0 \\
\hline ctz-aca/PVP & 500.1 & 139.0 \\
\hline ctz-aca/PVP & 500.1 & 41.6 \\
\hline ctz-aca/MCC & 500.1 & 416.0 \\
\hline ctz-aca/MCC & 500.1 & 125.0 \\
\hline ctz-ppa/PVP & 501.5 & 402.0 \\
\hline ctz-ppa/PVP & 501.5 & 134.0 \\
\hline ctz-ppa/PVP & 501.5 & 40.2 \\
\hline ctz-ppa/MCC & 501.5 & 402.0 \\
\hline ctz-ppa/PVP & 501.5 & 134.0 \\
\hline ctz-ppa/PVP & 501.5 & 40.2 \\
\hline$\left(\mathrm{bzamH}^{+}\right)\left(\mathrm{ctz}^{-}\right) / \mathrm{PVP}$ & 498.9 & 354.8 \\
\hline$\left(\mathrm{bzamH}^{+}\right)\left(\mathrm{ctz}^{-}\right) / \mathrm{MCC}$ & 498.9 & 354.8 \\
\hline htz-nia/PVP & 251.4 & 178.2 \\
\hline htz-nia/MCC & 251.4 & 178.2 \\
\hline htz-nia/HPC & 251.4 & 178.2 \\
\hline htz-nia/ $\alpha$-lactose & 251.4 & 178.2 \\
\hline htz-nia/NaTC & 251.4 & 178.2 \\
\hline htz-nia/DA & 251.4 & 178.2 \\
\hline
\end{tabular}

a To each sample $50 \mu \mathrm{L}$ acetonitrile was added. 
Table S3. Formation of the cocrystals in the presence of excipients. Composition of samples.

\begin{tabular}{|c|c|c|c|}
\hline sample & mg API & mg coformer & mg excipient \\
\hline ctz/bipy/PVP & 328.2 & 86.6 & 328.2 \\
\hline ctz/bipy/MCC & 328.2 & 86.6 & 328.2 \\
\hline ctz/ebipy/PVP & 310.5 & 191.3 & 310.5 \\
\hline ctz/ebipy/MCC & 310.5 & 191.3 & 310.5 \\
\hline ctz/pbipy/PVP & 295.7 & 198.2 & 295.7 \\
\hline ctz/pbipy/MCC & 295.7 & 198.2 & 295.7 \\
\hline ctz/pyr/PVP & 393.3 & 106.5 & 393.3 \\
\hline ctz/pyr/MCC & 393.3 & 106.5 & 393.3 \\
\hline ctz/hyp/PVP & 378.5 & 121.7 & 378.5 \\
\hline ctz/hyp/MCC & 378.5 & 121.7 & 378.5 \\
\hline ctz/hma/PVP & 340.0 & 161.2 & 340.0 \\
\hline ctz/hma/MCC & 340.0 & 161.2 & 340.0 \\
\hline ctz/bza/PVP & 354.8 & 145.3 & 354.8 \\
\hline ctz/bza/MCC & 354.8 & 145.3 & 354.8 \\
\hline ctz/ina/PVP & 354.8 & 146.5 & 354.8 \\
\hline ctz/ina/MCC & 354.8 & 146.5 & 354.8 \\
\hline ctz/nia/PVP & 354.8 & 146.4 & 354.8 \\
\hline ctz/nia/MCC & 354.8 & 146.4 & 354.8 \\
\hline ctz/nia/HPC & 354.8 & 146.4 & 354.8 \\
\hline ctz/nia/ $\alpha$-lactose & 354.8 & 146.4 & 354.8 \\
\hline ctz/nia/NaTC & 354.8 & 146.4 & 354.8 \\
\hline ctz/nia/DA & 354.8 & 146.4 & 354.8 \\
\hline ctz/cbz/PVP & 277.9 & 444.1 & 277.9 \\
\hline ctz/cbz/MCC & 277.9 & 444.1 & 277.9 \\
\hline ctz/cbz/HPC & 277.9 & 444.1 & 277.9 \\
\hline ctz/cbz/ $\alpha$-lactose & 277.9 & 444.1 & 277.9 \\
\hline ctz/cbz/NaTC & 277.9 & 444.1 & 277.9 \\
\hline $\mathrm{ctz} / \mathrm{cbz} / \mathrm{DA}$ & 277.9 & 444.1 & 277.9 \\
\hline ctz/aca/PVP & 416.9 & 83.2 & 41.6 \\
\hline ctz/aca/MCC & 416.9 & 83.2 & 125.0 \\
\hline ctz/ppa/PVP & 402.1 & 99.4 & 40.2 \\
\hline ctz/ppa/MCC & 402.1 & 99.4 & 40.2 \\
\hline ctz/bzam/PVP & 354.8 & 144.1 & 354.8 \\
\hline ctz/bzam/MCC & 354.8 & 144.1 & 354.8 \\
\hline htz/nia/PVP & 178.2 & 73.2 & 178.2 \\
\hline htz/nia/MCC & 178.2 & 73.2 & 178.2 \\
\hline htz/nia/HPC & 178.2 & 73.2 & 178.2 \\
\hline htz/nia/ $\alpha$-lactose & 178.2 & 73.2 & 178.2 \\
\hline htz/nia/NaTC & 178.2 & 73.2 & 178.2 \\
\hline htz/nia/DA & 178.2 & 73.2 & 178.2 \\
\hline
\end{tabular}

${ }^{\text {a }}$ To each sample $50 \mu \mathrm{L}$ acetonitrile was added. 
Table S4. IR bands $\left(\mathrm{cm}^{-1}\right)$ of the coformers and of the coformers after milling with PVP (ctz : PVP 1:1 weight ratio)

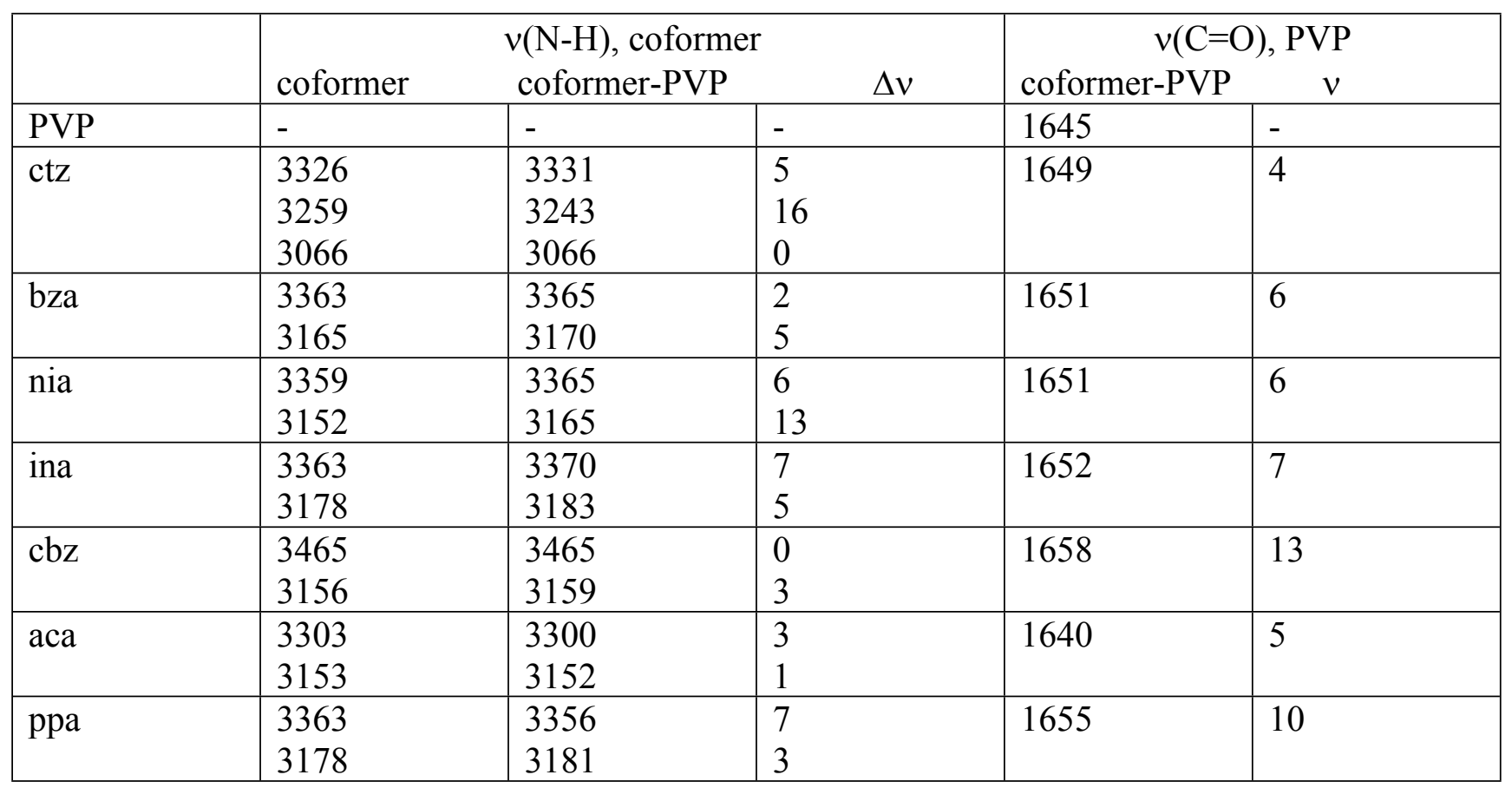

Table S5. Characteristic IR band $\left(\mathrm{cm}^{-1}\right)$ of $\mathrm{MCC}$ after milling with the respective coformer $(1: 1$ weight ratio)

\begin{tabular}{|l|l|}
\hline MCC & 1023, br; 1051, sh \\
\hline MCC/bza & $1026 ; 1056$ \\
\hline MCC/bzam & $1027 ; 1055$ \\
\hline MCC/bipy & $1032 ; 1056$ \\
\hline MCC/hma & $1047 ; 1064$ \\
\hline MCC/ppa & covered by ppa band \\
\hline
\end{tabular}


Table S6. IR bands $\left(\mathrm{cm}^{-1}\right)$ of ctz, htz, cbz and nia after milling with HPC, $\alpha$-lactose, DA and NaTC and after cocrystal formation (ctz : excipient 1:1 weight ratio).

\begin{tabular}{|c|c|c|c|c|}
\hline & $v(\mathrm{~N}-\mathrm{H})$ & $v(\mathrm{C}=\mathrm{O}), \mathrm{nia} / \mathrm{cbz}$ & $\Delta v(\mathrm{~N}-\mathrm{H})$ & $\Delta v(\mathrm{C}=\mathrm{O})$ \\
\hline ctz & $\begin{array}{l}3326 \\
3259 \\
3066\end{array}$ & & $\begin{array}{l}- \\
- \\
-\end{array}$ & \\
\hline ctz/HPC & $\begin{array}{l}3331 \\
3244 \\
3066\end{array}$ & & $\begin{array}{l}5 \\
15 \\
0 \\
\end{array}$ & \\
\hline ctz/ $\alpha$-lactose & $\begin{array}{l}3330 \\
3244 \\
3077\end{array}$ & & $\begin{array}{l}4 \\
15 \\
11 \\
\end{array}$ & \\
\hline ctz/DA & $\begin{array}{l}3332 \\
3245 \\
3066\end{array}$ & & $\begin{array}{l}6 \\
14 \\
0\end{array}$ & \\
\hline ctz/NaTC & $\begin{array}{l}3332 \\
3244 \\
3066\end{array}$ & & $\begin{array}{l}6 \\
15 \\
0\end{array}$ & \\
\hline nia & $\begin{array}{l}3359 \\
3152\end{array}$ & 1673 & $\begin{array}{l}- \\
- \\
\end{array}$ & - \\
\hline nia/HPC & $\begin{array}{l}3364 \\
3157\end{array}$ & 1677 & $\begin{array}{l}5 \\
5\end{array}$ & 4 \\
\hline nia/ $\alpha$-lactose & $\begin{array}{l}3361 \\
3158 \\
\end{array}$ & 1677 & $\begin{array}{l}2 \\
6 \\
\end{array}$ & 4 \\
\hline nia/DA & $\begin{array}{l}3364 \\
3159\end{array}$ & 1677 & $\begin{array}{l}5 \\
7\end{array}$ & 4 \\
\hline nia/NaTC & $\begin{array}{l}3364 \\
3160\end{array}$ & 1678 & $\begin{array}{l}5 \\
8 \\
\end{array}$ & 5 \\
\hline $\mathrm{cbz}$ & $\begin{array}{l}3464 \\
3156\end{array}$ & 1673 & - & - \\
\hline cbz/HPC & $\begin{array}{l}3465 \\
3159\end{array}$ & 1676 & $\begin{array}{l}1 \\
3 \\
\end{array}$ & 3 \\
\hline cbz/ $\alpha$-lactose & $\begin{array}{l}3464 \\
3159 \\
\end{array}$ & 1677 & $\begin{array}{l}0 \\
3 \\
\end{array}$ & 4 \\
\hline cbz/DA & 3485 & 1674 & 21 & 1 \\
\hline cbz/NaTC & 3465 & 1656 & 1 & 17 \\
\hline ctz-nia & $\begin{array}{l}3366 \\
3151\end{array}$ & 1652 & $\begin{array}{l}7 \\
1\end{array}$ & 21 \\
\hline htz-nia & 3375 & 1653 & 16 & 20 \\
\hline cbz-nia & $\begin{array}{l}3337 \\
3168\end{array}$ & 1668 & $\begin{array}{l}11 \\
12\end{array}$ & 5 \\
\hline
\end{tabular}




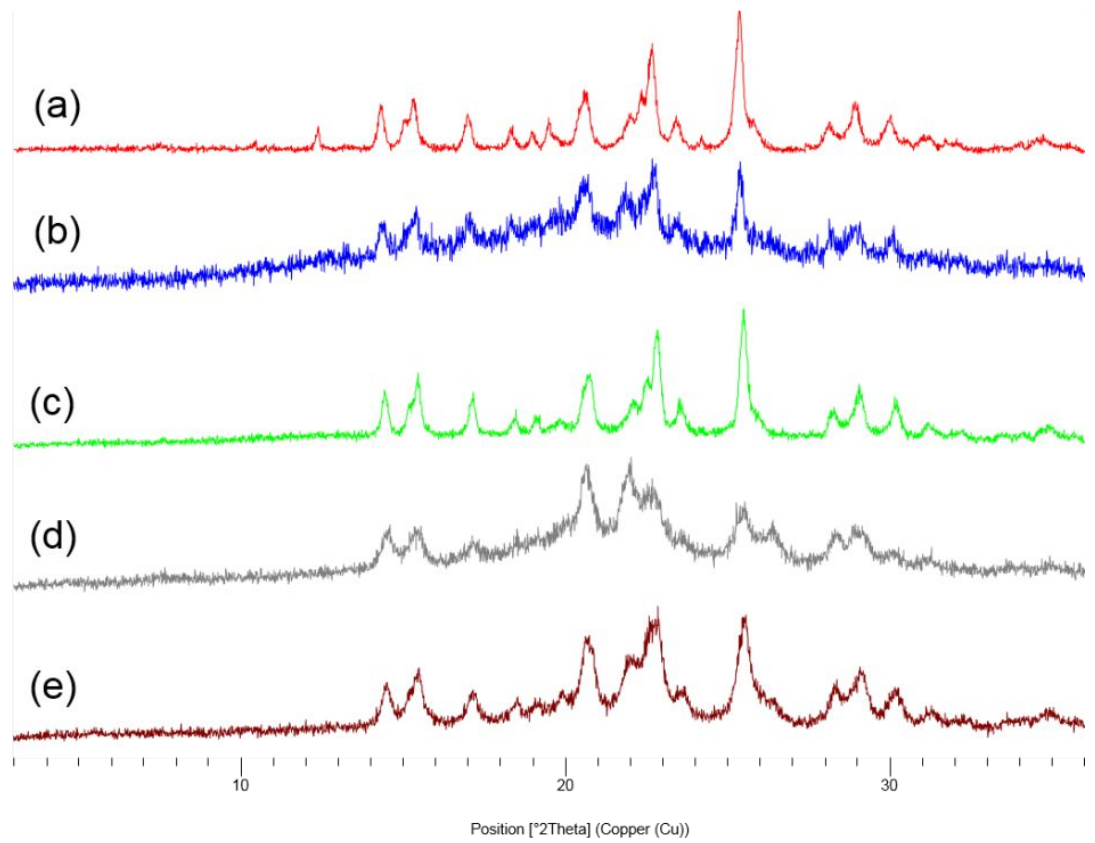

Figure S1. XRPD pattern of (a) the ctz-bipy cocrystal, (b) the ctz-bipy cocrystal after milling with PVP (ctz : PVP 1:1 w/w), (c) the ctz-bipy cocrystal after milling with PVP and after storage for $30 \mathrm{~d}$ at $20{ }^{\circ} \mathrm{C}, 56 \% \mathrm{RH}$, (d) the ctz-bipy cocrystal after milling with MCC (ctz : MCC 1:1 w/w) and (e) the ctz-bipy cocrystal after milling with MCC and after storage for $30 \mathrm{~d}$ at $20^{\circ} \mathrm{C}, 56 \% \mathrm{RH}$. 


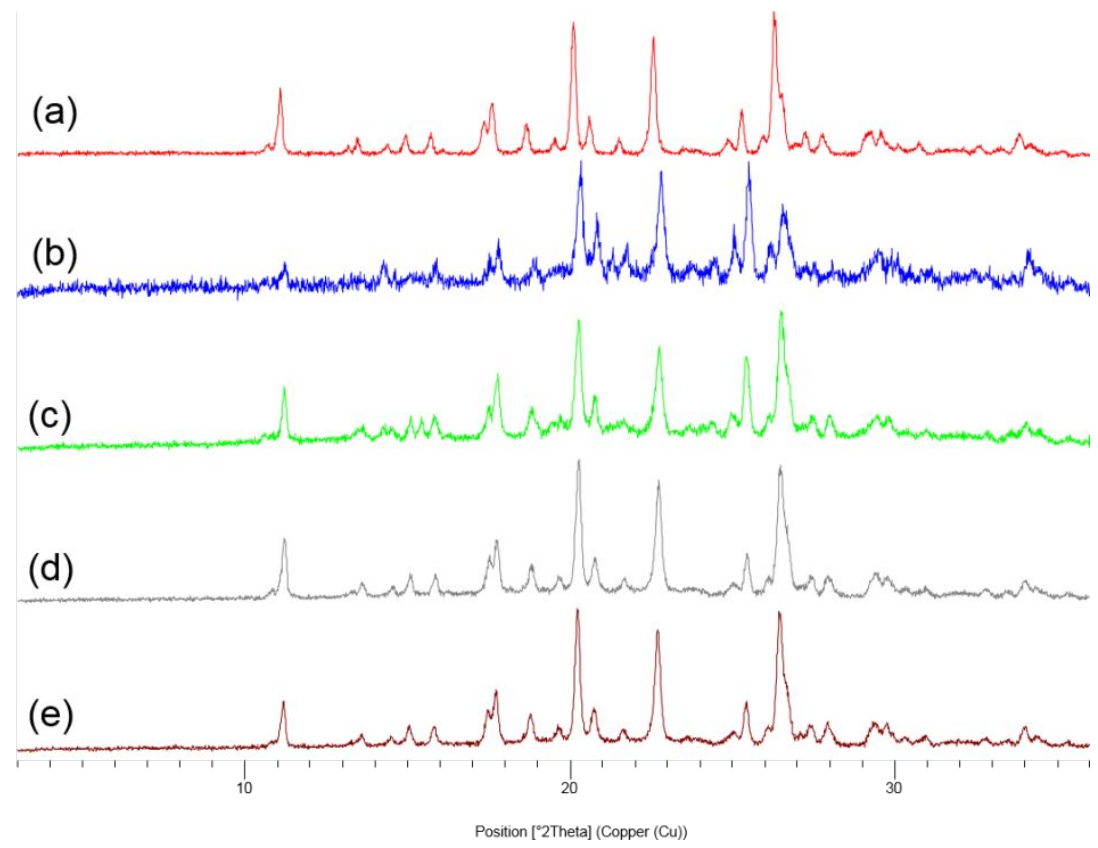

Figure S2. XRPD pattern of (a) the ctz-ebipy cocrystal, (b) the ctz-ebipy cocrystal after milling with PVP (ctz: PVP 1:1 w/w), (c) the ctz-ebipy cocrystal after milling with PVP and after storage for $30 \mathrm{~d}$ at $20{ }^{\circ} \mathrm{C}, 56 \% \mathrm{RH}$, (d) the ctz-ebipy cocrystal after milling with MCC (ctz : MCC 1:1 w/w) and (e) the ctz-ebipy cocrystal after milling with $\mathrm{MCC}$ and after storage for $30 \mathrm{~d}$ at $20{ }^{\circ} \mathrm{C}, 56 \% \mathrm{RH}$. 


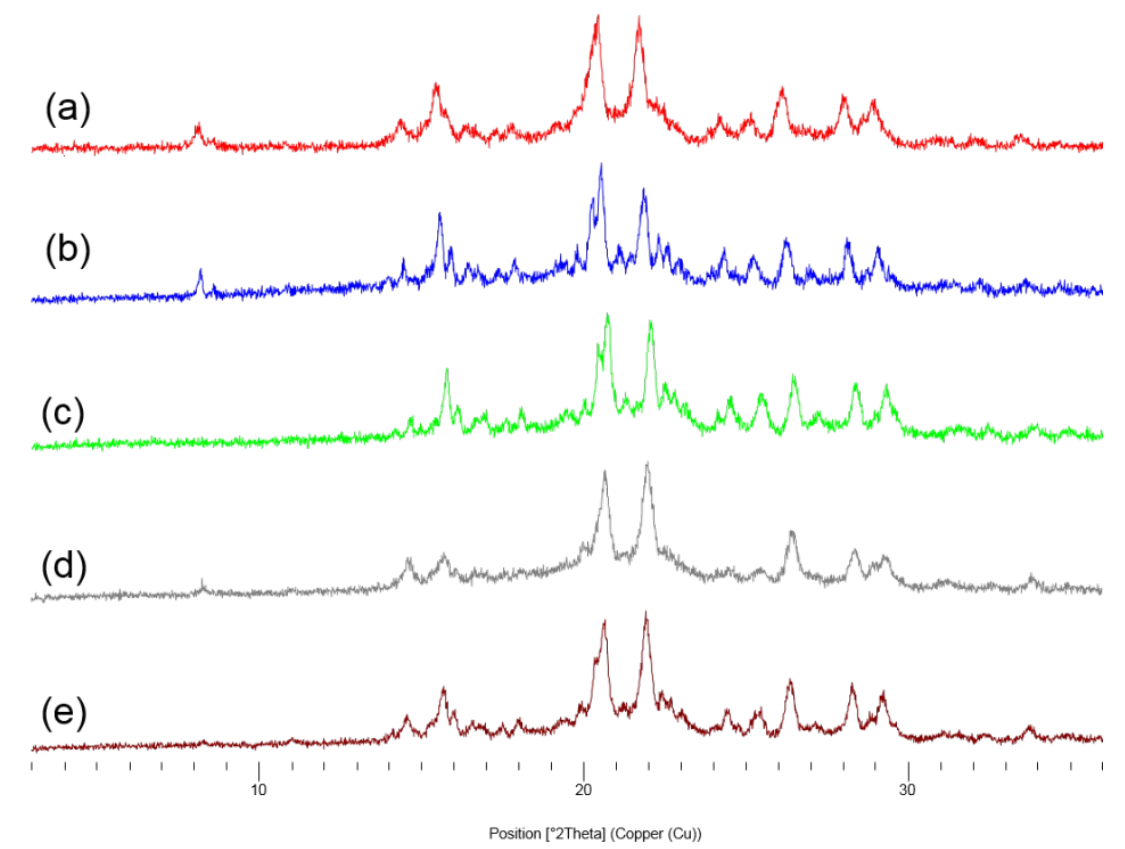

Figure S3. XRPD pattern of (a) the ctz-pbipy cocrystal, (b) the ctz-pbipy cocrystal after milling with PVP (ctz : PVP 1:1 w/w), (c) the ctz-pbipy cocrystal after milling with PVP and after storage for $30 \mathrm{~d}$ at $20^{\circ} \mathrm{C}, 56 \% \mathrm{RH}$, (d) the ctz-pbipy cocrystal after milling with MCC (ctz : MCC 1:1 w/w) and (e) the ctz-pbipy cocrystal after milling with $\mathrm{MCC}$ and after storage for $30 \mathrm{~d}$ at $20{ }^{\circ} \mathrm{C}, 56 \% \mathrm{RH}$. 


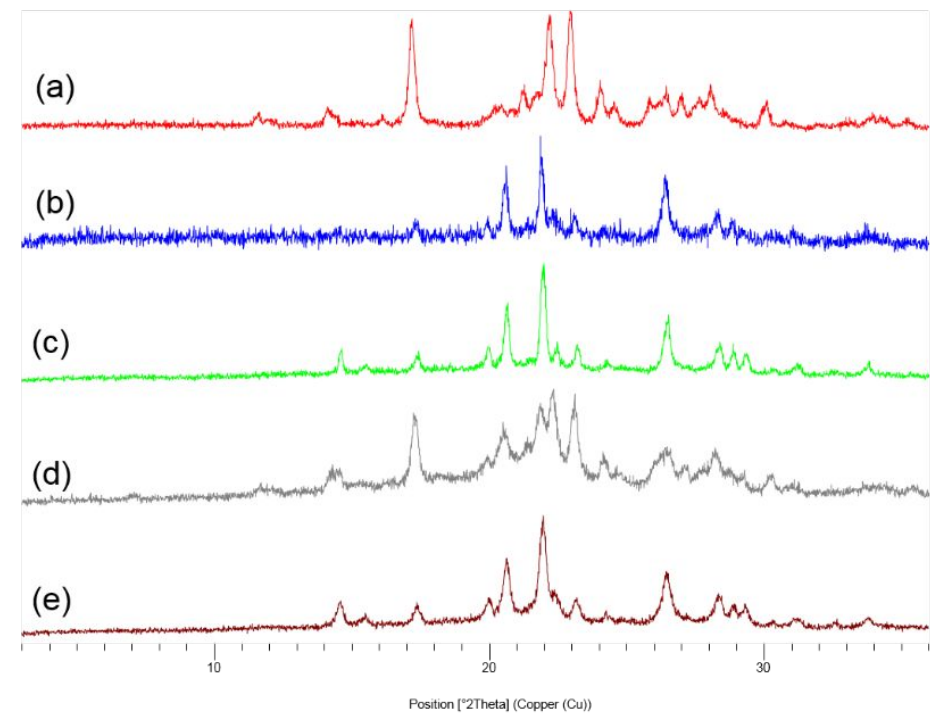

Figure S4. XRPD pattern of (a) the ctz-pyr cocrystal, (b) the ctz-pyr cocrystal after milling with PVP (ctz : PVP 1:1 w/w), (c) the ctz-pyr cocrystal after milling with PVP and after storage for $30 \mathrm{~d}$ at $20{ }^{\circ} \mathrm{C}, 56 \% \mathrm{RH}$, (d) the ctz-pyr cocrystal after milling with MCC (ctz : MCC 1:1 w/w) and (e) the ctz-pyr cocrystal after milling with MCC and after storage for $30 \mathrm{~d}$ at $20^{\circ} \mathrm{C}, 56 \% \mathrm{RH}$. 


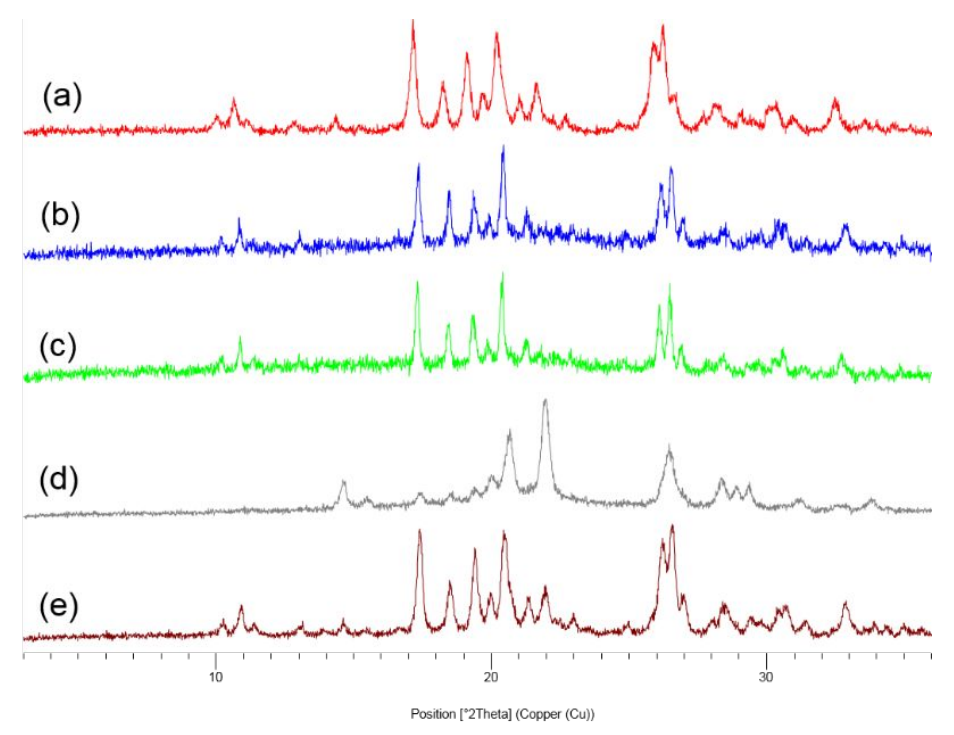

Figure S5. XRPD pattern of (a) the ctz-hyp cocrystal, (b) the ctz-hyp cocrystal after milling with PVP (ctz : PVP 1:1 w/w), (c) the ctz-hyp cocrystal after milling with PVP and after storage for $30 \mathrm{~d}$ at $20{ }^{\circ} \mathrm{C}, 56 \% \mathrm{RH}$, (d) the ctz-hyp cocrystal after milling with MCC (ctz : MCC 1:1 w/w) and (e) the ctz-hyp cocrystal after milling with MCC and after storage for $30 \mathrm{~d}$ at $20^{\circ} \mathrm{C}, 56 \% \mathrm{RH}$.

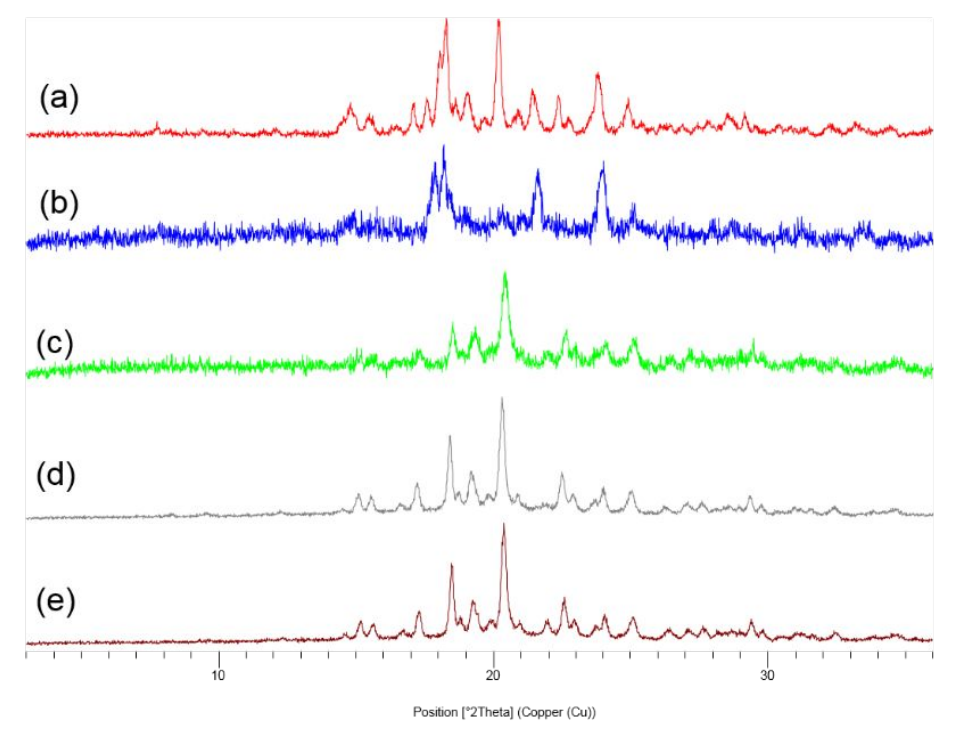

Figure S6. XRPD pattern of (a) the ctz-hma cocrystal, (b) the ctz-hma cocrystal after milling with PVP (ctz : PVP 1:1 w/w), (c) the ctz-hma cocrystal after milling with PVP and after storage for $30 \mathrm{~d}$ at $20{ }^{\circ} \mathrm{C}, 56 \% \mathrm{RH},(\mathrm{d})$ the ctz-hma cocrystal after milling with MCC (ctz : MCC 1:1 w/w) and (e) the ctz-hma cocrystal after milling with $\mathrm{MCC}$ and after storage for $30 \mathrm{~d}$ at $20^{\circ} \mathrm{C}, 56 \% \mathrm{RH}$. 


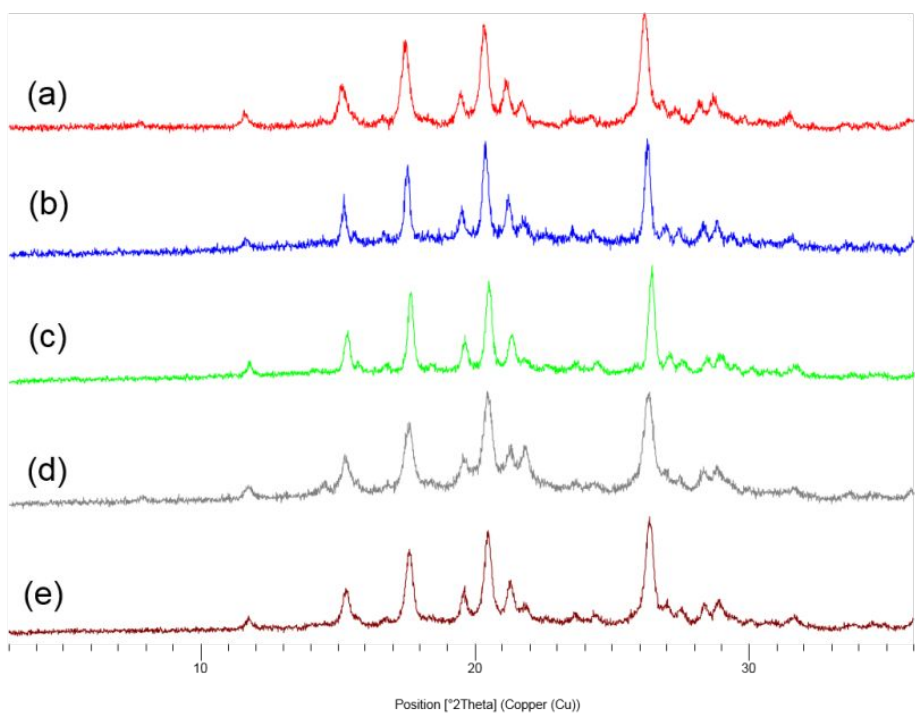

Figure S7. XRPD pattern of (a) the ctz-bza cocrystal, (b) the ctz-bza cocrystal after milling with PVP (ctz : PVP 1:1 w/w), (c) the ctz-bza cocrystal after milling with PVP and after storage for $30 \mathrm{~d}$ at $20{ }^{\circ} \mathrm{C}, 56 \% \mathrm{RH}$, (d) the ctz-bza cocrystal after milling with MCC (ctz : MCC 1:1 w/w) and (e) the ctz-bza cocrystal after milling with MCC and after storage for $30 \mathrm{~d}$ at $20^{\circ} \mathrm{C}, 56 \% \mathrm{RH}$. 


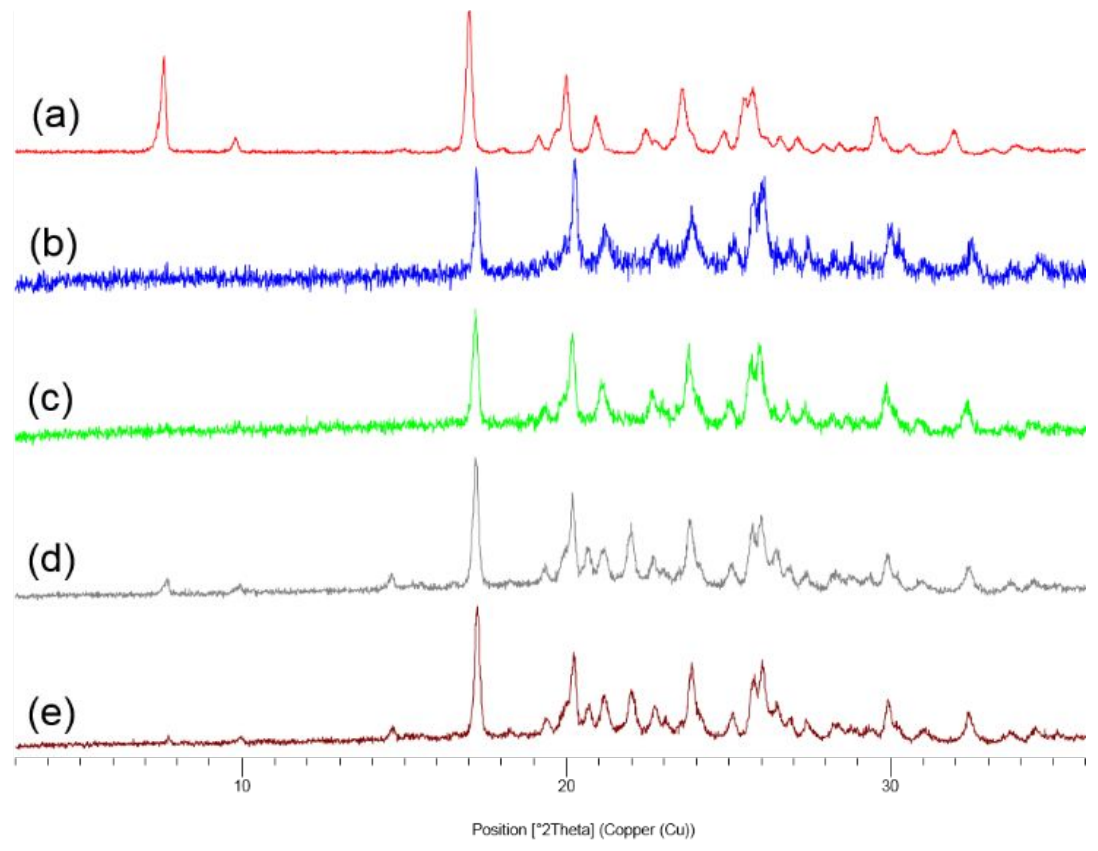

Figure S8. XRPD pattern of (a) the ctz-nia cocrystal, (b) the ctz-nia cocrystal after milling with PVP (ctz: PVP 1:1 w/w), (c) the ctz-nia cocrystal after milling with PVP and after storage for $30 \mathrm{~d}$ at $20{ }^{\circ} \mathrm{C}, 56 \% \mathrm{RH},(\mathrm{d})$ the ctz-nia cocrystal after milling with MCC (ctz: MCC 1:1 w/w) and (e) the ctz-nia cocrystal after milling with $\mathrm{MCC}$ and after storage for $30 \mathrm{~d}$ at $20{ }^{\circ} \mathrm{C}, 56 \% \mathrm{RH}$. 


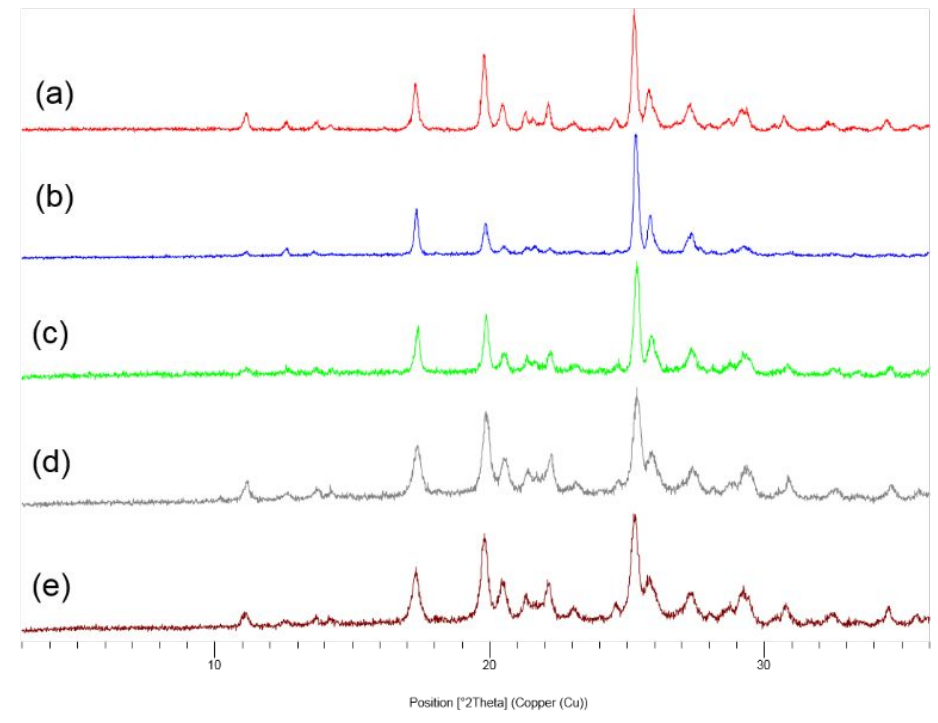

Figure S9. XRPD pattern of (a) the ctz-ina cocrystal, (b) the ctz-ina cocrystal after milling with PVP (ctz: PVP 1:1 w/w), (c) the ctz-ina cocrystal after milling with PVP and after storage for $30 \mathrm{~d}$ at $20{ }^{\circ} \mathrm{C}, 56 \% \mathrm{RH},(\mathrm{d})$ the ctz-ina cocrystal after milling with MCC (ctz: MCC 1:1 w/w) and (e) the ctz-ina cocrystal after milling with $\mathrm{MCC}$ and after storage for $30 \mathrm{~d}$ at $20{ }^{\circ} \mathrm{C}, 56 \% \mathrm{RH}$. 

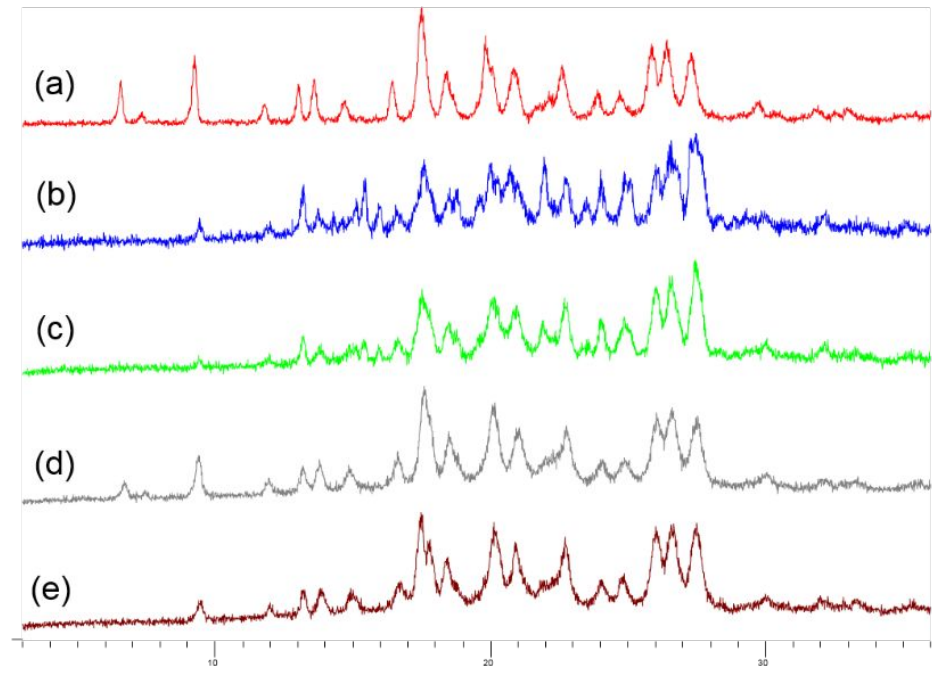

Figure S10. XRPD pattern of (a) the ctz-cbz cocrystal, (b) the ctz-cbz cocrystal after milling with PVP (ctz : PVP 1:1 w/w), (c) the ctz-cbz cocrystal after milling with PVP and after storage for $30 \mathrm{~d}$ at $20{ }^{\circ} \mathrm{C}, 56 \% \mathrm{RH}$, (d) the ctz-cbz cocrystal after milling with MCC (ctz : MCC 1:1 w/w) and (e) the ctz-cbz cocrystal after milling with MCC and after storage for $30 \mathrm{~d}$ at $20^{\circ} \mathrm{C}, 56 \% \mathrm{RH}$. 


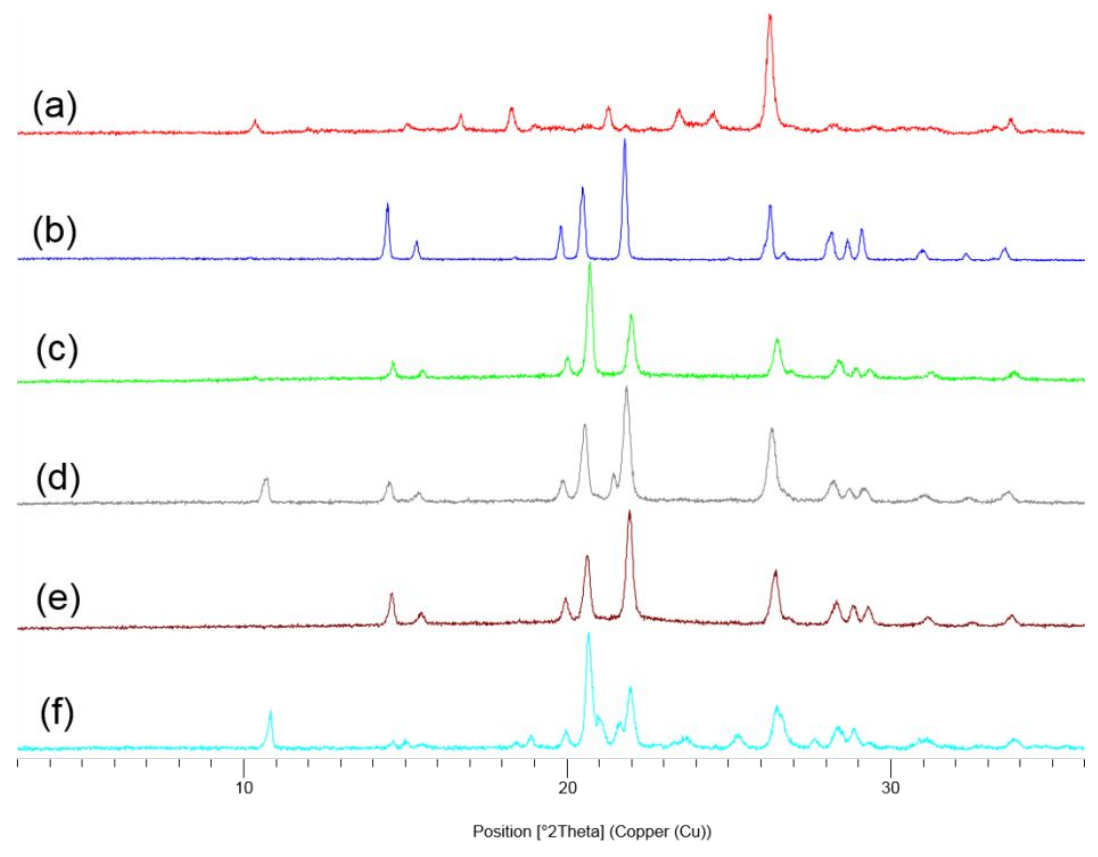

Figure S11. XRPD pattern of (a) the ctz-ppa cocrystal, (b) ctz, (c) the ctz-ppa cocrystal after milling with PVP (ctz : PVP 1:1 w/w), (d) the ctz-ppa cocrystal after milling with PVP (ctz : PVP 10:1 w/w), (e) the ctz-ppa cocrystal after milling with MCC (ctz : MCC 1:1 w/w) and (f) the ctz-ppa cocrystal after milling with MCC (ctz : MCC 10:1 w/w). 


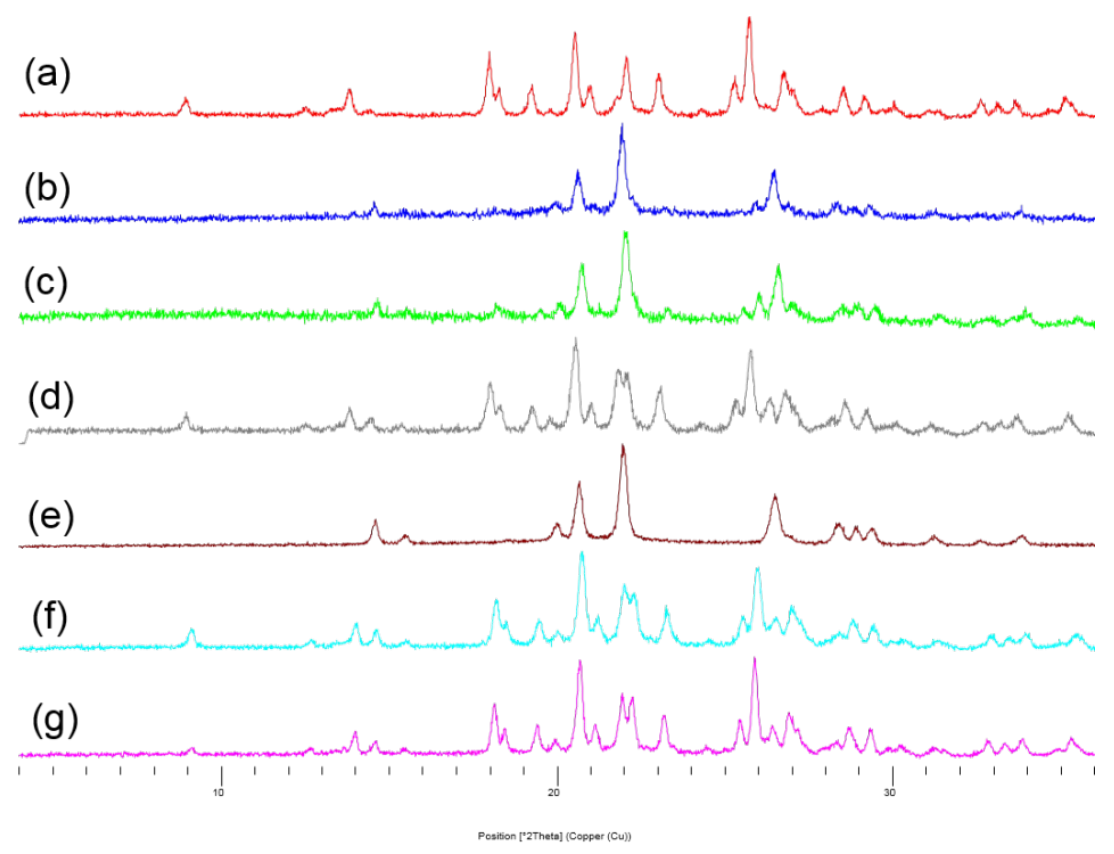

Figure S12. XRPD pattern of (a) the ctz-aca cocrystal, (b) the ctz-aca cocrystal after milling with PVP (ctz : PVP 1:1 w/w), (c) the ctz-aca cocrystal after milling with PVP (ctz : PVP 3:1 w/w), (d) the ctz-aca cocrystal after milling with PVP (ctz : PVP 10:1 w/w), (e) the ctz-aca cocrystal after milling with MCC (ctz : MCC 1:1 w/w), (f) the ctz-aca cocrystal after milling with MCC (ctz : MCC 3:1 w/w) and (g) the ctz-aca cocrystal after milling with MCC (3:1 w/w) and after storage for $30 \mathrm{~d}$ at $20{ }^{\circ} \mathrm{C}, 56 \% \mathrm{RH}$. 


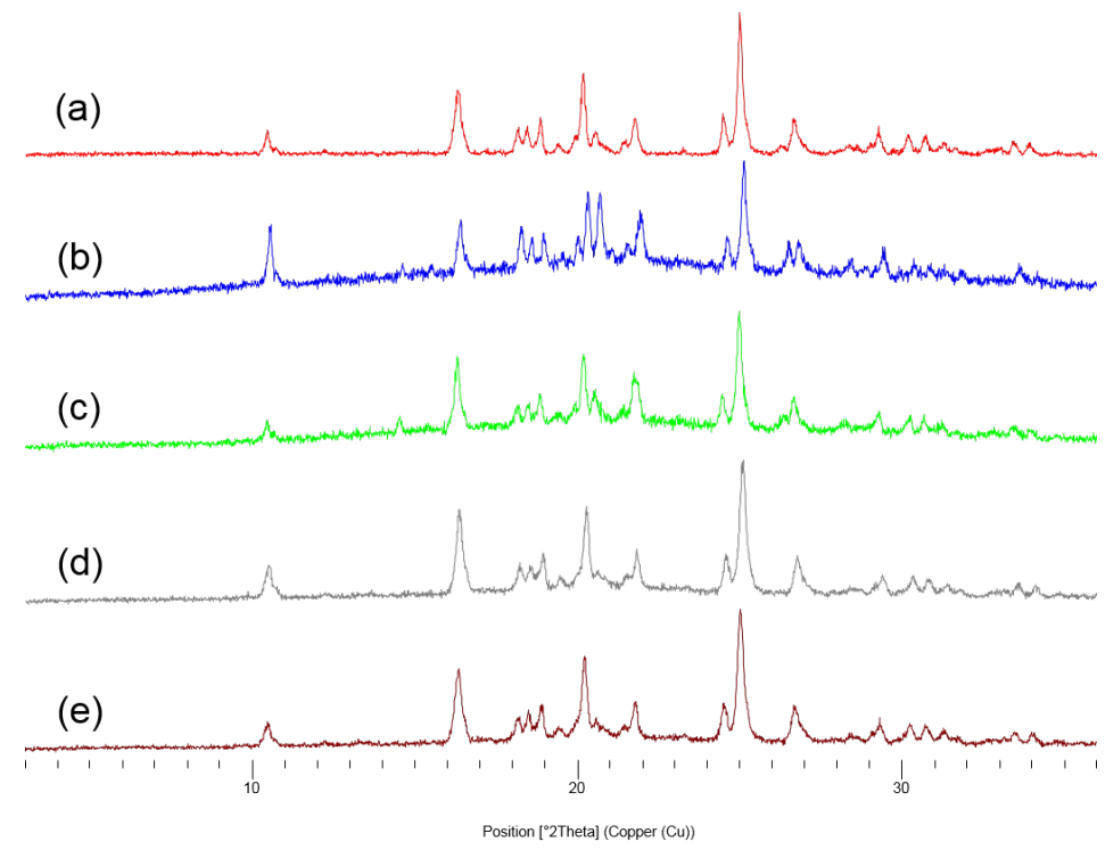

Figure S13. XRPD pattern of (a) $\left(\right.$ bzamH $\left.^{+}\right)\left(\right.$ctz $\left.^{-}\right)$, (b) $\left(\right.$bzamH $\left.^{+}\right)\left(\right.$ctz $\left.^{-}\right)$after milling with PVP (bzam : PVP 1:1 w/w), (c) $\left(\mathrm{bzamH}^{+}\right)\left(\mathrm{ctz}^{-}\right)$after milling with PVP and after storage for $30 \mathrm{~d}$ at $20{ }^{\circ} \mathrm{C}, 56 \%$ RH, (d) $\left(\right.$ bzamH $\left.^{+}\right)\left(\mathrm{ctz}^{-}\right)$after milling with MCC (bzam : MCC 1:1 w/w) and (e) $\left(\right.$bzamH $\left.^{+}\right)\left(\mathrm{ctz}^{-}\right)$after milling with $\mathrm{MCC}$ and after storage for $30 \mathrm{~d}$ at $20{ }^{\circ} \mathrm{C}, 56 \% \mathrm{RH}$. 


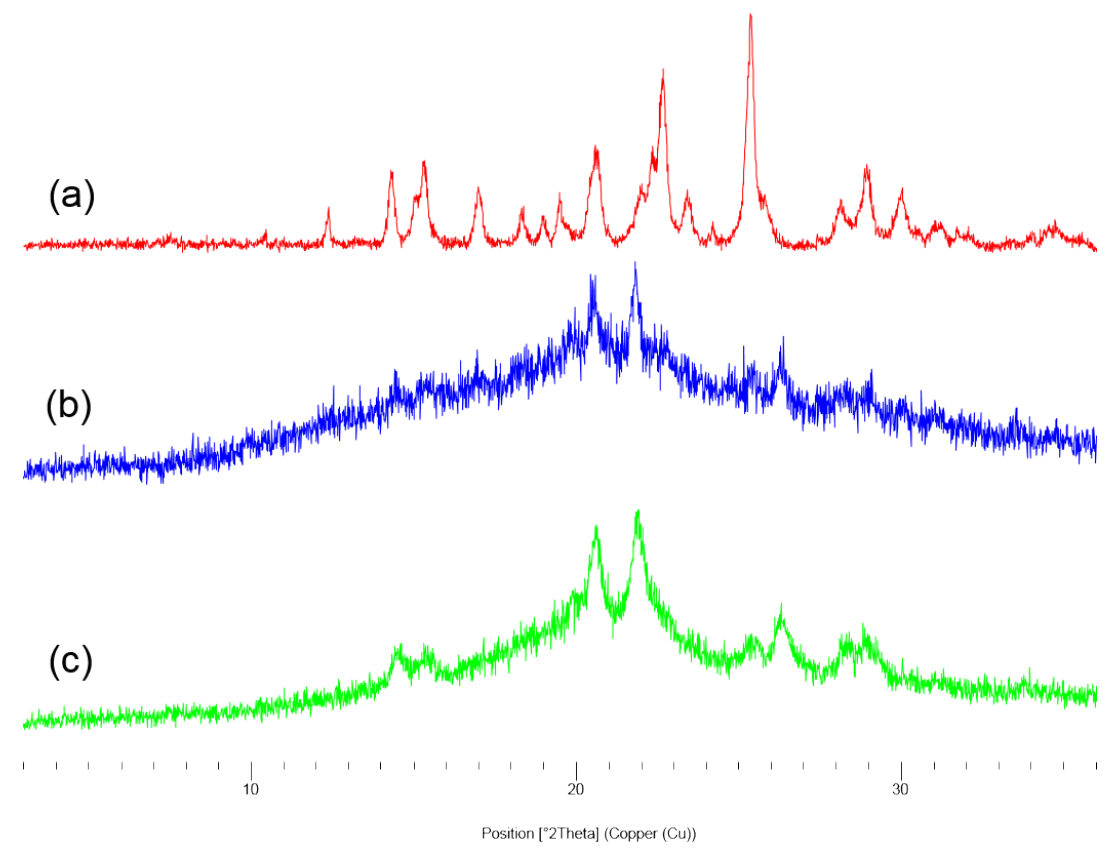

Figure S14. XRPD pattern of (a) the ctz-bipy cocrystal, (b) a milled mixture of ctz, bipy and PVP (ctz : bipy $=1: 0.5$ molar ratio; $c$ ctz $: \mathrm{PVP}=1: 1.5(\mathrm{w} / \mathrm{w})$ ) and $(\mathrm{c})$ a milled mixture of ctz, bipy and MCC (ctz : bipy = 1:0.5 molar ratio; ctz : $\mathrm{MCC}=1: 1.5(\mathrm{w} / \mathrm{w}))$.

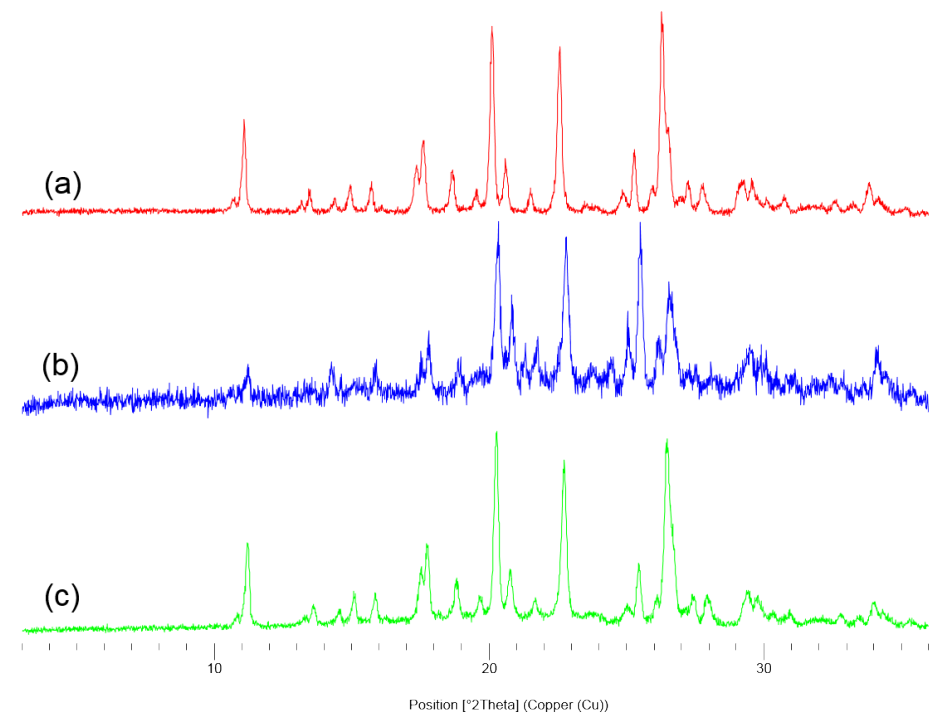

Figure S15. XRPD pattern of (a) the ctz-ebipy cocrystal, (b) a milled mixture of ctz, ebipy and PVP $(\mathrm{ctz}:$ ebipy $=1: 1$ molar ratio; ctz $:$ PVP $=1: 1(\mathrm{w} / \mathrm{w})$ ), and (c) a milled mixture of ctz, ebipy and $\operatorname{MCC}($ ctz $:$ ebipy $=1: 1$ molar ratio; $\operatorname{ctz}: \operatorname{MCC}=1: 1(\mathrm{w} / \mathrm{w}))$. 


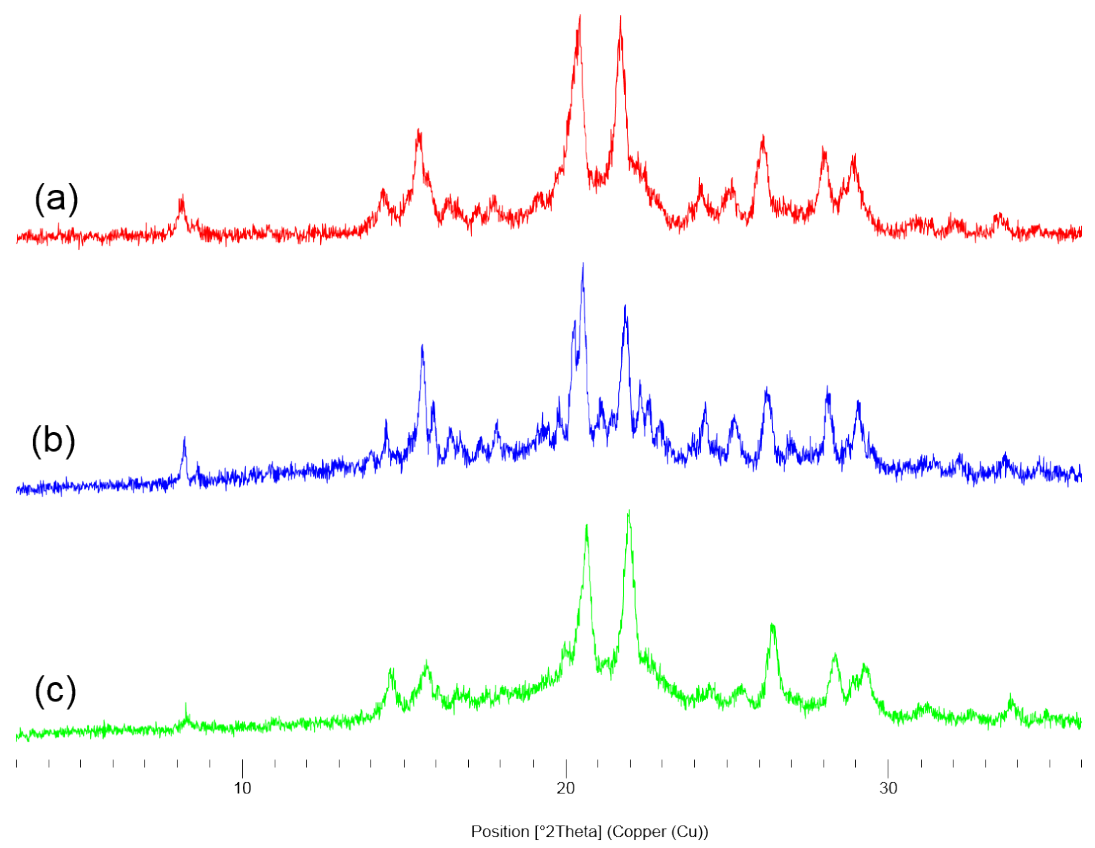

Figure S16. XRPD pattern of (a) the ctz-pbipy cocrystal, (b) a milled mixture of ctz, pbipy and PVP (ctz : pbipy = 1:2 molar ratio; ctz : $\mathrm{PVP}=1: 1(\mathrm{w} / \mathrm{w})$ ) and (c) a milled mixture of ctz, pbipy and MCC (ctz : pbipy = 1:2 molar ratio; ctz : $\mathrm{MCC}=1: 1(\mathrm{w} / \mathrm{w}))$.

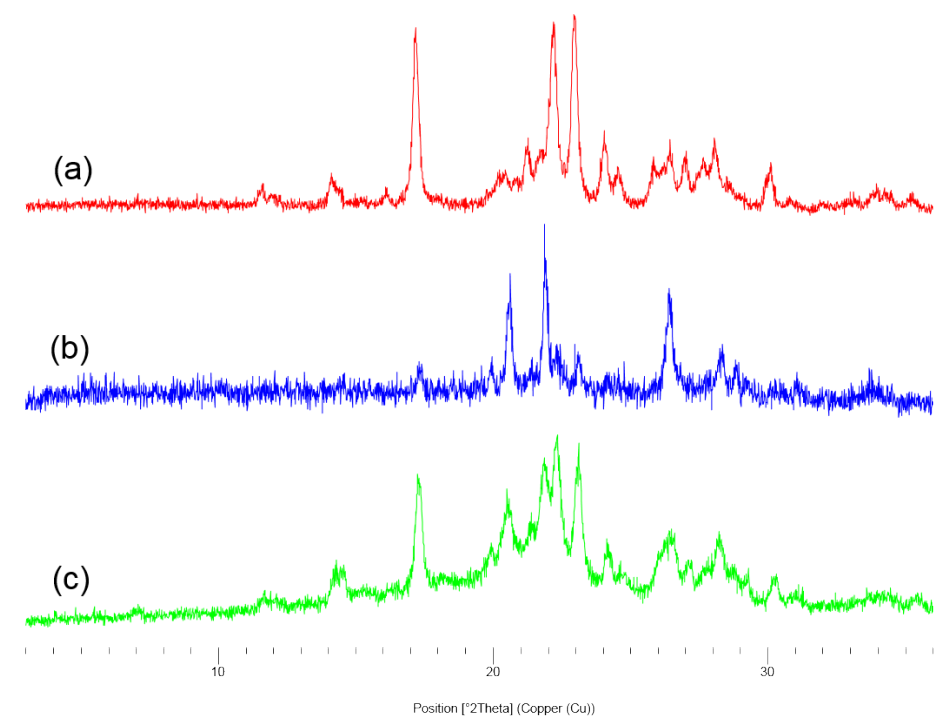

Figure S17. XRPD pattern of (a) the ctz-pyr cocrystal, (b) a milled mixture of ctz, pyr and PVP (ctz $:$ pyr $=1: 2$ molar ratio; ctz : $\mathrm{PVP}=1: 1(\mathrm{w} / \mathrm{w}))$ and $(\mathrm{c})$ a milled mixture of ctz, pyr and MCC (ctz : pyr $=1: 1$ molar ratio; $\mathrm{ctz}: \mathrm{MCC}=1: 1(\mathrm{w} / \mathrm{w}))$. 


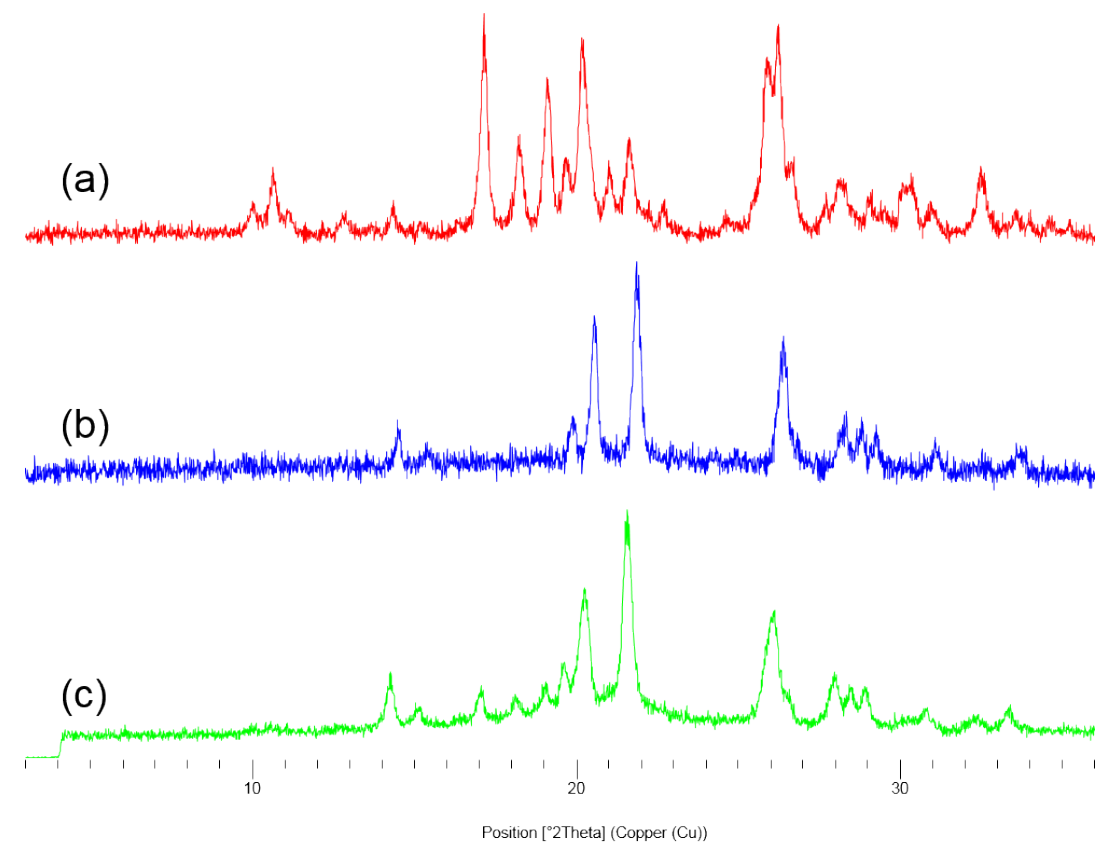

Figure S18. XRPD pattern of (a) the ctz-hyp cocrystal, (b) a milled mixture of ctz, hyp and PVP (ctz : hyp = 1:1 molar ratio; ctz : $\mathrm{PVP}=1: 1(\mathrm{w} / \mathrm{w})$ ) and (c) a milled mixture of ctz, hyp and MCC (ctz : hyp = 1:1 molar ratio; ctz : MCC =1:1(w/w)).

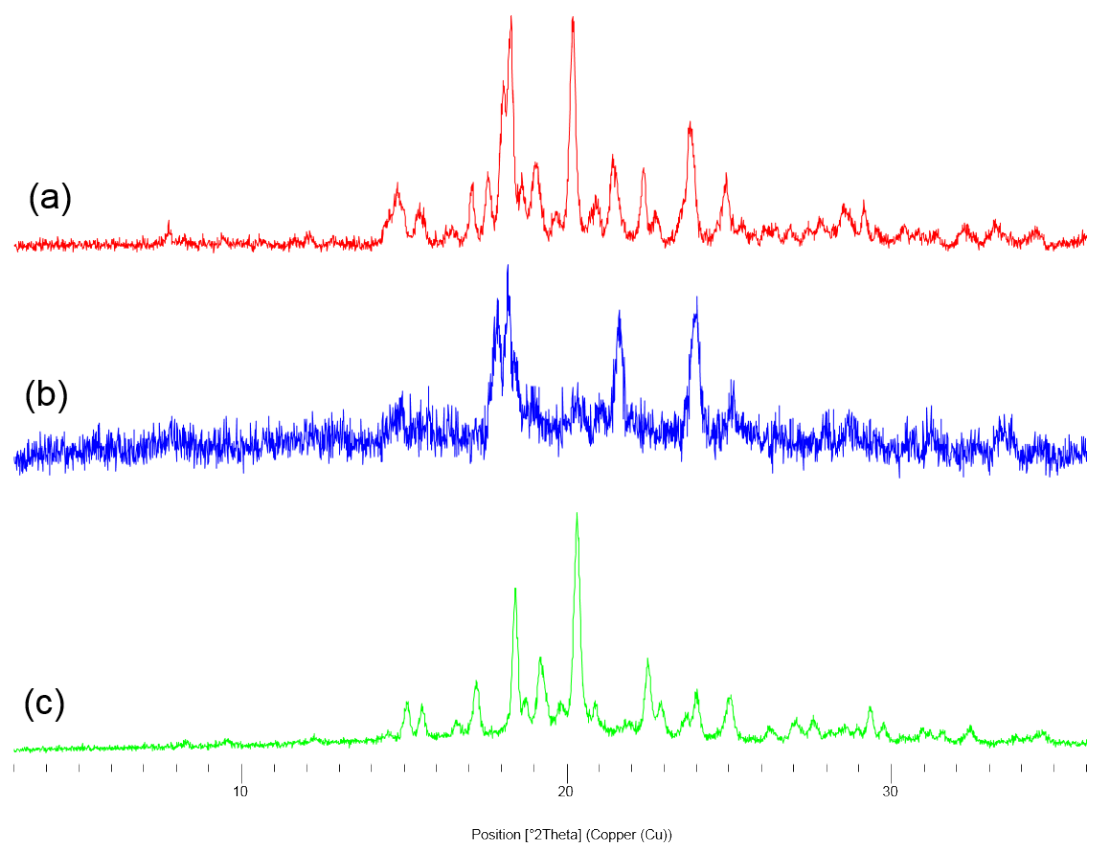

Figure S19. XRPD pattern of (a) the ctz-hma cocrystal, (b) a milled mixture of ctz, hma and PVP (ctz : hma $=1: 1$ molar ratio; $\mathrm{ctz}: \mathrm{PVP}=1: 1(\mathrm{w} / \mathrm{w})$ ) and (c) a milled mixture of ctz, hma and MCC $(\mathrm{ctz}: \mathrm{hma}=1: 1$ molar ratio; $\mathrm{ctz}: \mathrm{MCC}=1: 1(\mathrm{w} / \mathrm{w}))$. 


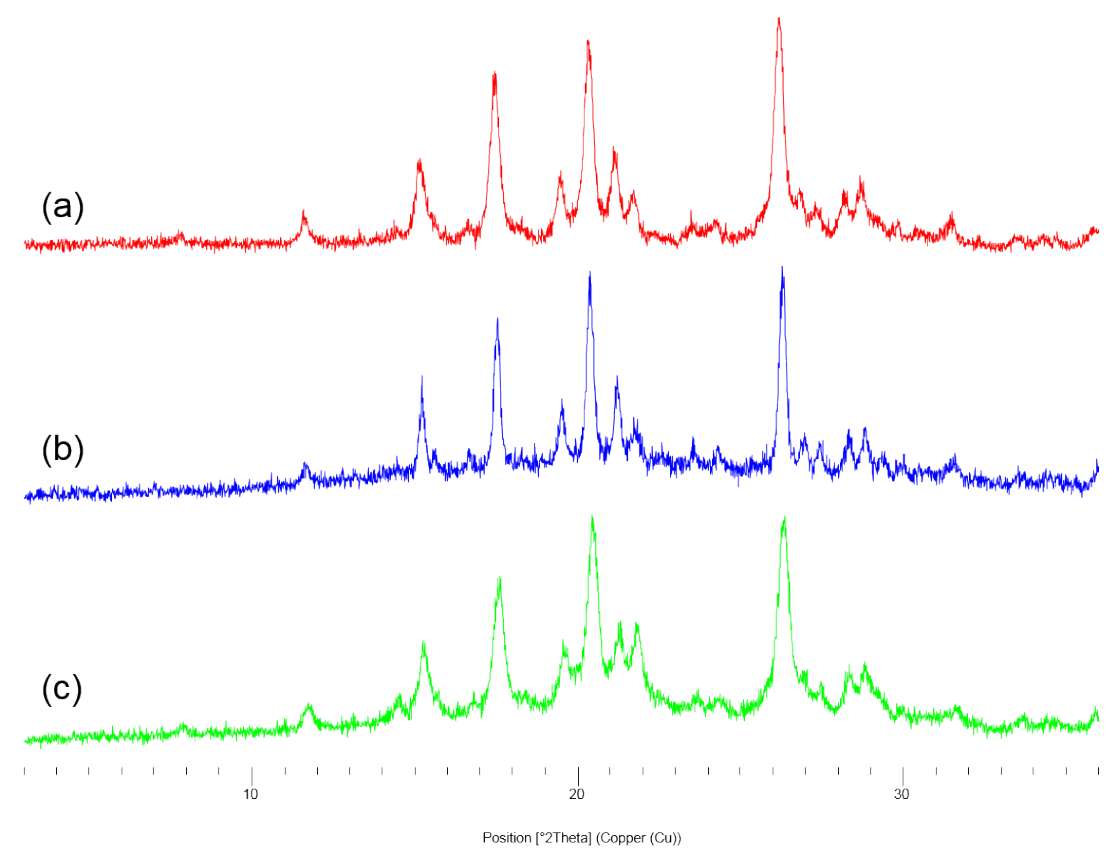

Figure S20. XRPD pattern of (a) the ctz-bza cocrystal, (b) a milled mixture of ctz, bza and PVP (ctz $:$ bza $=1: 1$ molar ratio; ctz $:$ PVP $=1: 1(\mathrm{w} / \mathrm{w})$ ) and (c) a milled mixture of ctz, bza and MCC (ctz : bza $=1: 1$ molar ratio; $\mathrm{ctz}: \mathrm{MCC}=1: 1(\mathrm{w} / \mathrm{w}))$.

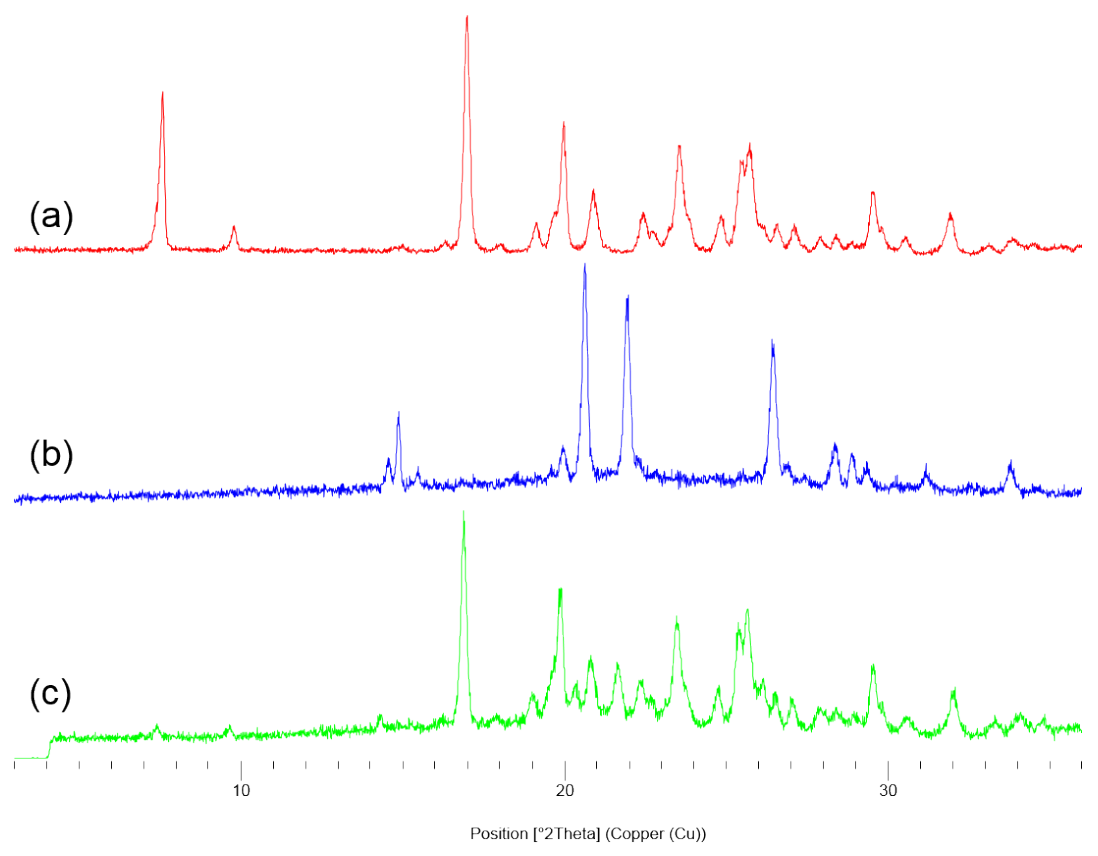

Figure S21. XRPD pattern of (a) ctz-nia, (b) a milled mixture of ctz, nia and PVP (ctz : nia $=1: 1$ molar ratio; ctz : PVP = 1:1 (w/w)) and (c) a milled mixture of ctz, nia and $\mathrm{MCC}(\mathrm{ctz}:$ nia $=1: 1$ molar ratio; ctz : $\mathrm{MCC}=1: 1(\mathrm{w} / \mathrm{w}))$. 


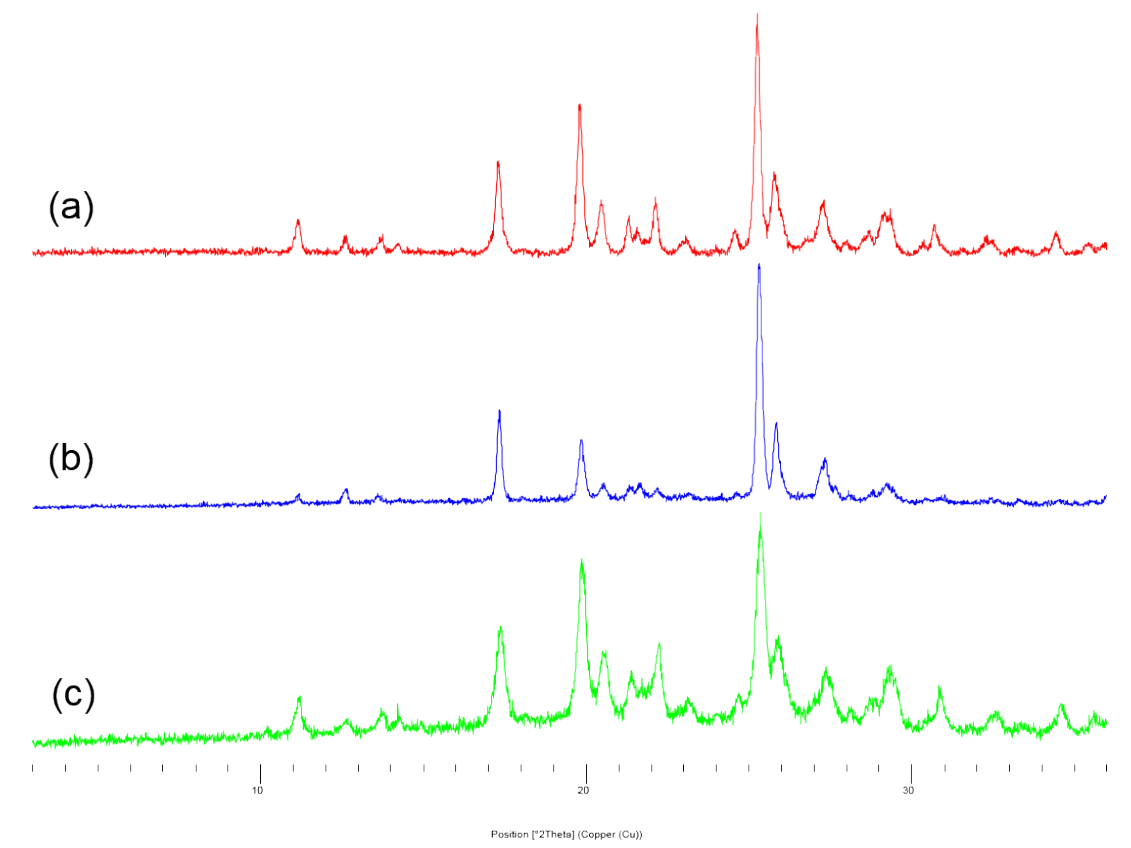

Figure S22. XRPD pattern of (a) ctz-ina, (b) a milled mixture of ctz, ina and PVP (ctz : ina $=1: 1$ molar ratio; ctz : PVP = 1:1 (w/w)) and (c) a milled mixture of ctz, ina and MCC (ctz : ina $=1: 1$ molar ratio; ctz : $\mathrm{MCC}=1: 1(\mathrm{w} / \mathrm{w}))$.

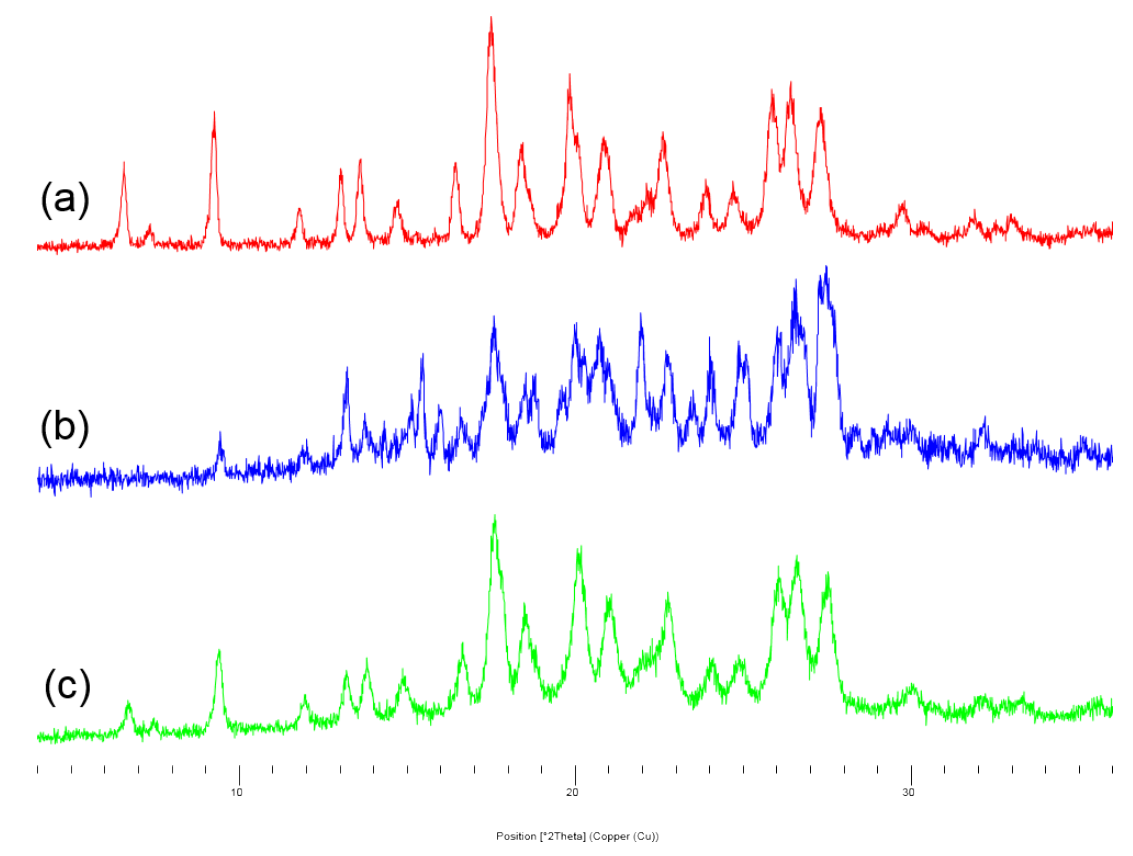

Figure S23. XRPD pattern of (a) ctz-cbz, (b) a milled mixture of ctz, cbz and PVP (ctz : cbz = 1:1 molar ratio; ctz : PVP = 1:1 (w/w)) and (c) a milled mixture of ctz, cbz and MCC (ctz : cbz = 1:1 molar ratio; ctz : $\mathrm{MCC}=1: 1(\mathrm{w} / \mathrm{w}))$. 

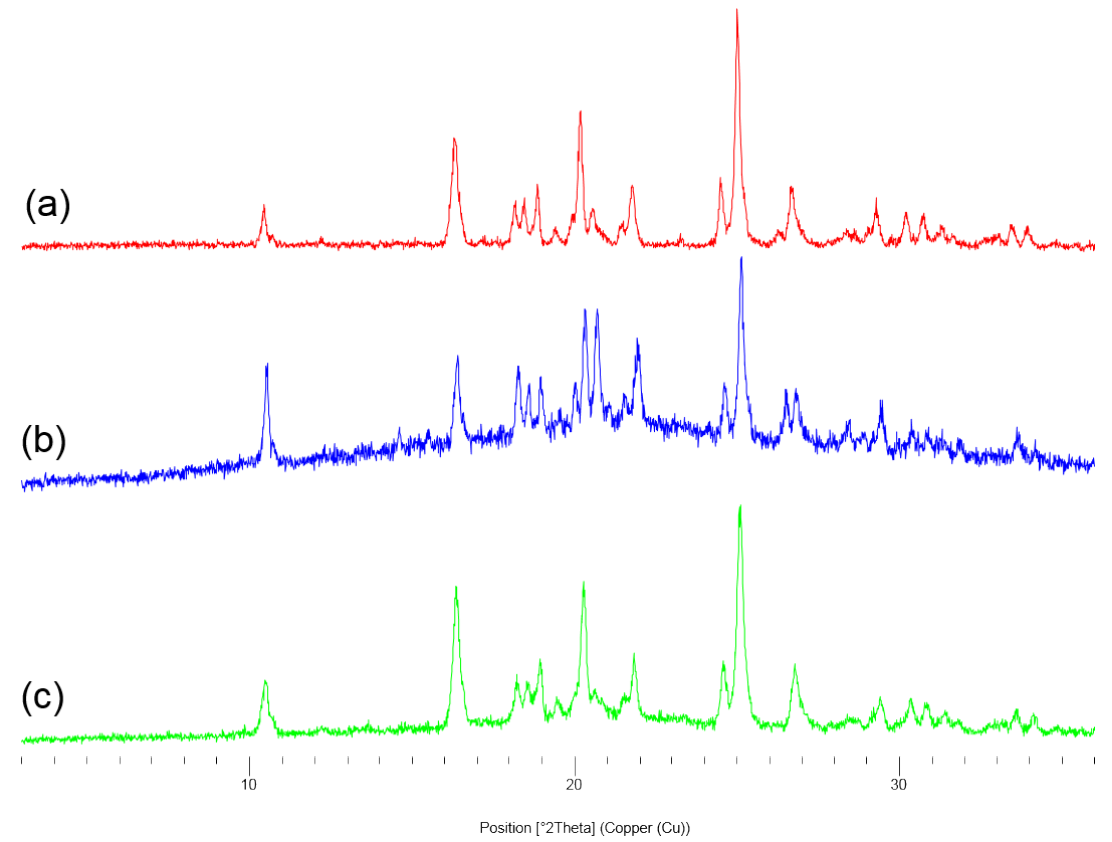

Figure S24. XRPD pattern of (a) $\left(\mathrm{bzamH}^{+}\right)\left(\mathrm{ctz}^{-}\right)$, (b) a milled mixture of ctz, bzam and PVP (ctz : bzam $=1: 1$ molar ratio; $c$ $:$ PVP $=1: 1(\mathrm{w} / \mathrm{w})$ ) and (c) a milled mixture of ctz, bzam and MCC (ctz : bzam $=1: 1$ molar ratio; ctz $: \mathrm{MCC}=1: 1(\mathrm{w} / \mathrm{w}))$. 


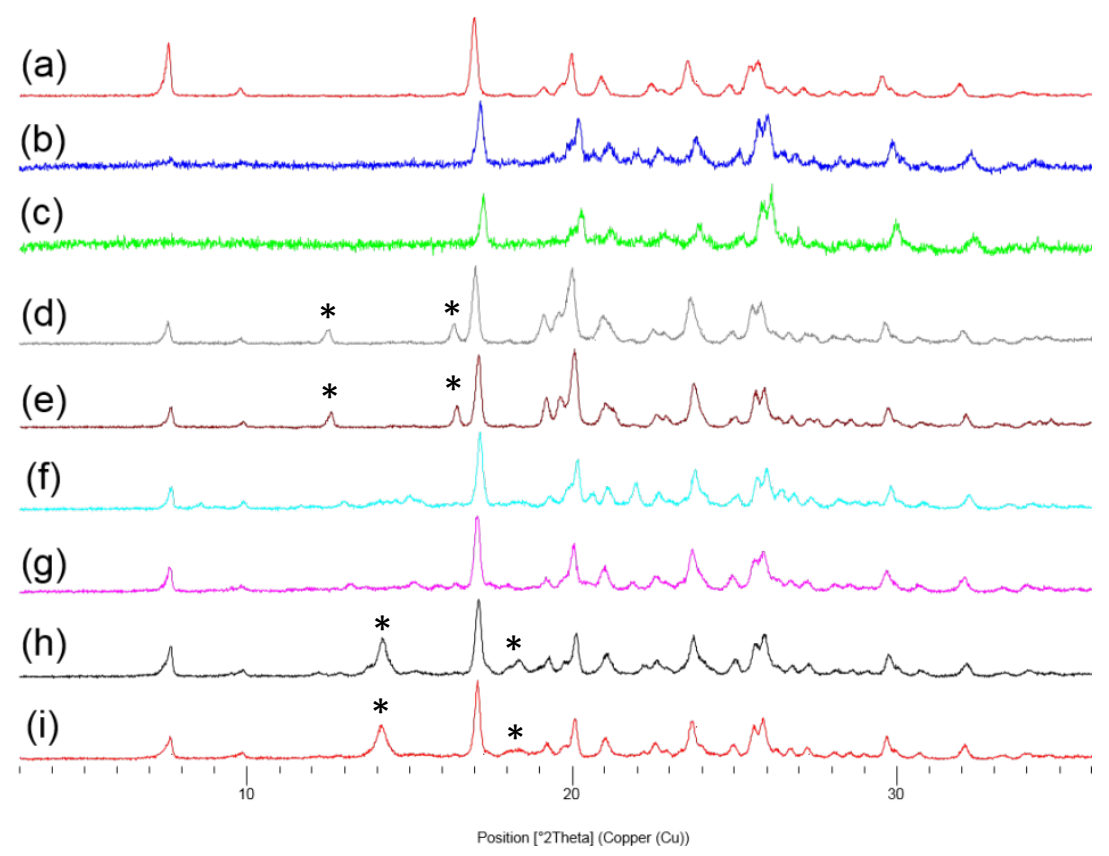

Figure S25. XRPD pattern of (a) the ctz-nia cocrystal, (b) the ctz-nia cocrystal after milling with HPC (ctz : PVP 1:1 w/w), (c) the ctz-nia cocrystal after milling with HPC and after storage for $30 \mathrm{~d}$ at $20{ }^{\circ} \mathrm{C}, 56 \% \mathrm{RH}$, (d) the ctz-nia cocrystal after milling with $\alpha$-lactose (ctz : $\alpha$-lactose $1: 1 \mathrm{w} / \mathrm{w}$ ), (e) the ctz-nia cocrystal after milling with $\alpha$-lactose and after storage for $30 \mathrm{~d}$ at $20{ }^{\circ} \mathrm{C}, 56 \% \mathrm{RH}$, (f) the ctz-nia cocrystal after milling with NaTC (ctz: NaTC 1:1 w/w), (g) the ctz-nia cocrystal after milling with NaTC and after storage for $30 \mathrm{~d}$ at $20{ }^{\circ} \mathrm{C}, 56 \% \mathrm{RH}$, (h) the ctz-nia cocrystal after milling with DA (ctz: DA 1:1 w/w) and (i) the ctz-nia cocrystal after milling with DA and after storage for $30 \mathrm{~d}$ at $20{ }^{\circ} \mathrm{C}, 56 \% \mathrm{RH} . *$ excipient peaks 


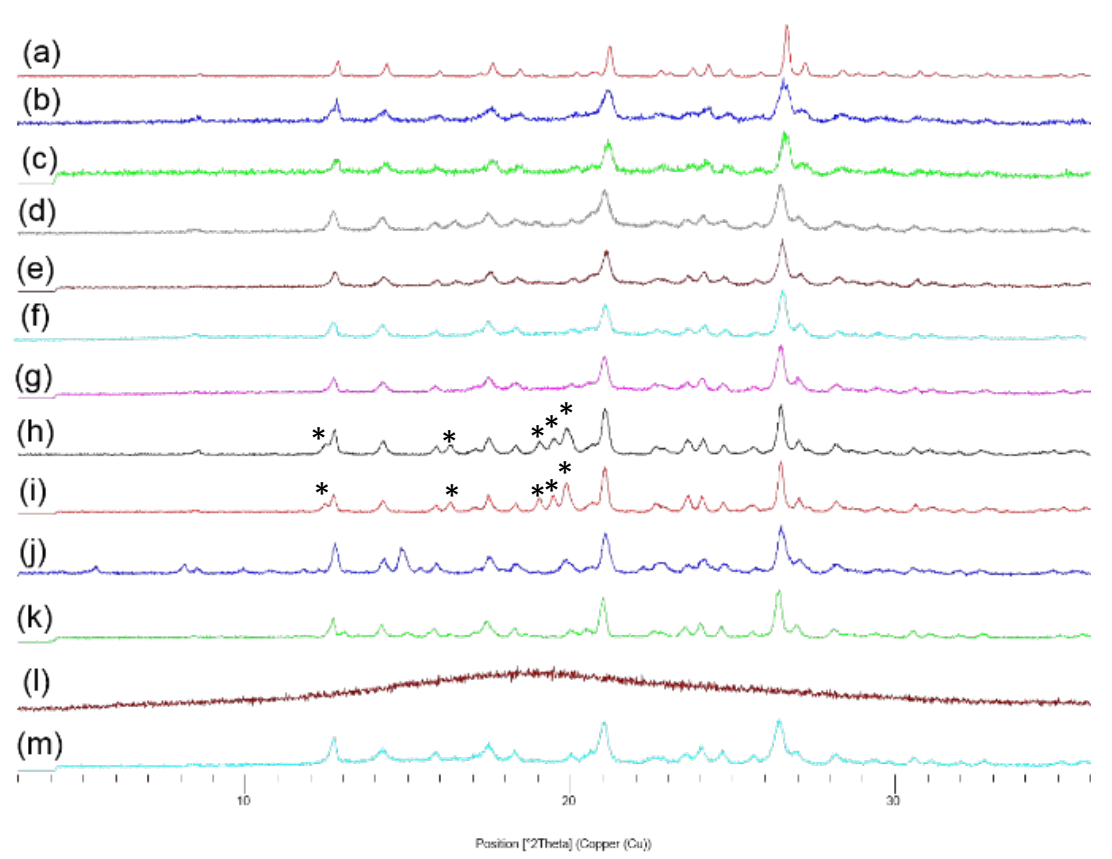

Figure S26. XRPD pattern of (a) the htz-nia cocrystal, (b) the htz-nia cocrystal after milling with PVP (htz : PVP 1:1 w/w), (c) the htz-nia cocrystal after milling with PVP and after storage for $30 \mathrm{~d}$ at $20{ }^{\circ} \mathrm{C}, 56 \% \mathrm{RH}$, (d) the htz-nia cocrystal after milling with MCC (htz : MCC 1:1 w/w), (e) the htz-nia cocrystal after milling with MCC and after storage for $30 \mathrm{~d}$ at $20^{\circ} \mathrm{C}, 56 \% \mathrm{RH}$, (f) the htznia cocrystal after milling with HPC (ctz: HPC 1:1 w/w), (g) the htz-nia cocrystal after milling with $\mathrm{HPC}$ and after storage for $30 \mathrm{~d}$ at $20^{\circ} \mathrm{C}, 56 \% \mathrm{RH}$, (h) the htz-nia cocrystal after milling with $\alpha$ lactose (htz : $\alpha$-lactose $1: 1 \mathrm{w} / \mathrm{w}$ ), (i) the htz-nia cocrystal after milling with $\alpha$-lactose and after storage for $30 \mathrm{~d}$ at $20^{\circ} \mathrm{C}, 56 \% \mathrm{RH},(\mathrm{j})$ the htz-nia cocrystal after milling with NaTC (htz : NaTC $1: 1 \mathrm{w} / \mathrm{w}),(\mathrm{k})$ the htz-nia cocrystal after milling with NaTC and after storage for $30 \mathrm{~d}$ at $20{ }^{\circ} \mathrm{C}, 56 \%$ $\mathrm{RH}$, (l) the htz-nia cocrystal after milling with DA (htz : DA 1:1 w/w) and (m) the htz-nia cocrystal after milling with DA and after storage for $30 \mathrm{~d}$ at $20^{\circ} \mathrm{C}, 56 \% \mathrm{RH}$. * excipient peaks 

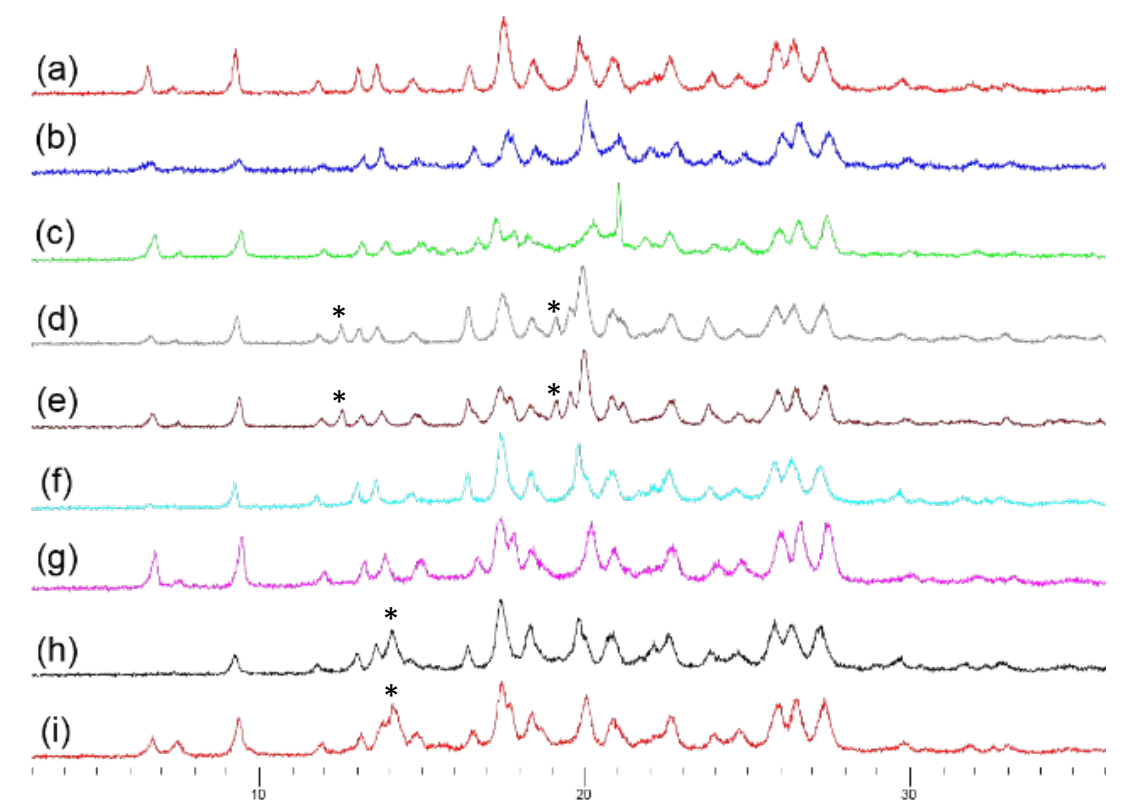

Postion ['2Theta] (Coppor (Cu))

Figure S27. XRPD pattern of (a) the ctz-cbz cocrystal, (b) the ctz-cbz cocrystal after milling with HPC (ctz : HPC 1:1 w/w), (c) the ctz-cbz cocrystal after milling with HPC and after storage for $30 \mathrm{~d}$ at $20{ }^{\circ} \mathrm{C}, 56 \% \mathrm{RH}$, (d) the ctz-cbz cocrystal after milling with $\alpha$-lactose (ctz: $\alpha$-lactose $1: 1 \mathrm{w} / \mathrm{w}$ ), (e) the ctz-cbz cocrystal after milling with $\alpha$-lactose and after storage for $30 \mathrm{~d}$ at $20{ }^{\circ} \mathrm{C}, 56 \% \mathrm{RH}$, (f) the ctz-cbz cocrystal after milling with NaTC (ctz: NaTC 1:1 w/w), (g) the ctz-cbz cocrystal after milling with NaTC and after storage for $30 \mathrm{~d}$ at $20{ }^{\circ} \mathrm{C}, 56 \% \mathrm{RH}$, (h) the ctz-cbz cocrystal after milling with DA (ctz: DA 1:1 w/w) and (i) the ctz-cbz cocrystal after milling with DA and after storage for $30 \mathrm{~d}$ at $20{ }^{\circ} \mathrm{C}, 56 \% \mathrm{RH}$. * excipient peaks 


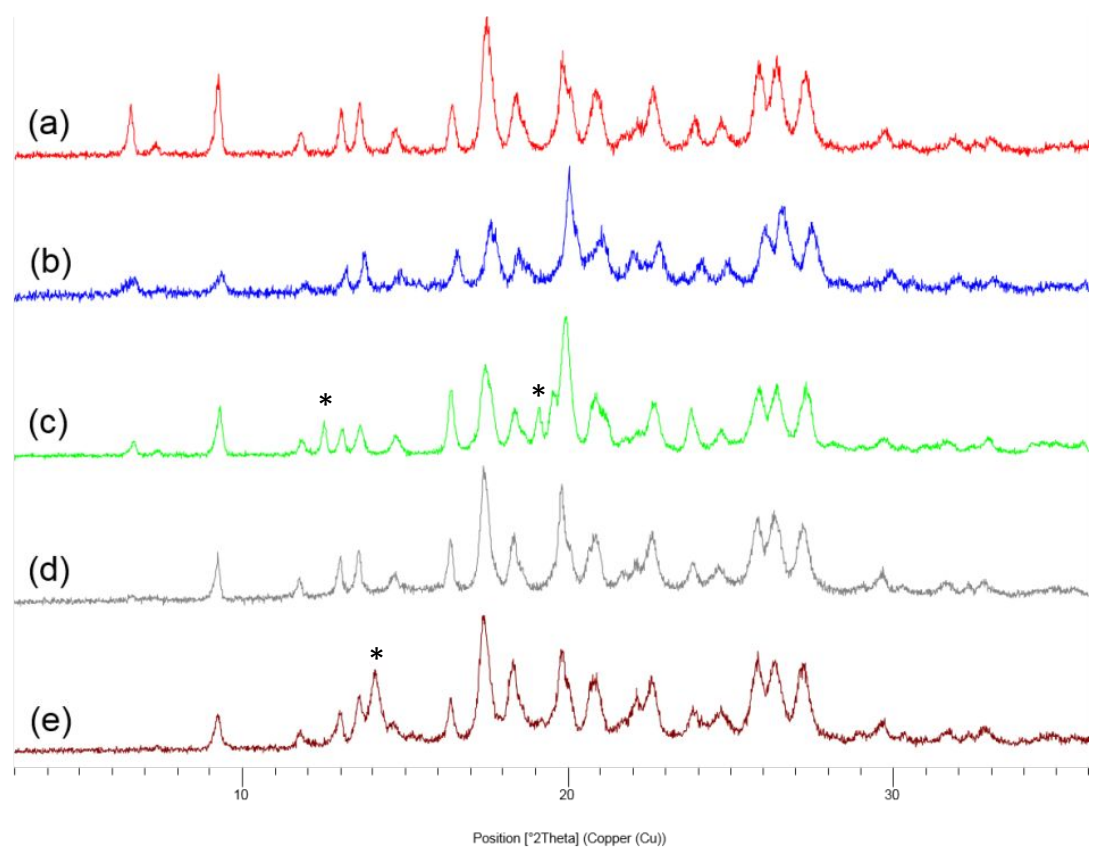

Figure S28. XRPD pattern of (a) the ctz-cbz cocrystal, (b) a milled mixture of ctz, cbz and HPC (ctz : HPC 1:1 w/w), (c) a milled mixture of ctz, cbz and $\alpha$-lactose (ctz : $\alpha$-lactose 1:1 w/w), (d) a milled mixture of ctz, cbz and NaTC (ctz : NaTC 1:1 w/w) and (e) a milled mixture of ctz, cbz and DA (ctz : DA 1:1 w/w). * excipient peaks 